\title{
Clinical efficacy and biomarker analysis of neoadjuvant atezolizumab in operable urothelial carcinoma in the ABACUS trial
}

Thomas Powles ${ }^{1 *}$, Mark Kockx², Alejo Rodriguez-Vida ${ }^{3}$, Ignacio Duran ${ }^{4}$, Simon J. Crabb ${ }^{5}$, Michiel S. Van Der Heijden ${ }^{6}$, Bernadett Szabados ${ }^{1}$, Albert Font Pous ${ }^{7}$, Gwenaelle Gravis ${ }^{8}$, Urbano Anido Herranz ${ }^{9}$, Andrew Protheroe ${ }^{10}$, Alain Ravaud ${ }^{11}$, Denis Maillet ${ }^{12}$, Maria Jose Mendez ${ }^{13}$, Cristina Suarez ${ }^{14}$, Mark Linch $^{15}$, Aaron Prendergast ${ }^{1}$, Pieter-Jan van Dam², Diana Stanoeva ${ }^{2}$, Sofie Daelemans ${ }^{16}$, Sanjeev Mariathasan ${ }^{17}$, Joy S Tea ${ }^{17}$, Kelly Mousa ${ }^{1}$, Romain Banchereau ${ }^{17 \alpha}$, Daniel Castellano ${ }^{18 \alpha}$

${ }^{*}$ Corresponding author: Professor Thomas Powles MBBS MRCP MD, Director of Bart Cancer Centre; Queen Mary University of London, Centre for Experimental Cancer Medicine, Barts Cancer Institute, Old Anatomy Building, Charterhouse Square, London EC1M 6BQ, UK | Tel: +44 (0)20 78828498 | Email:

t.powles@qmul.ac.uk

${ }^{\alpha}$ Contributed equally to the work

Key words: Urothelial bladder cancer, atezolizumab, PD-L1, biomarkers, pathological complete response, TGF $\beta$, TMB, FGF.

${ }^{1}$ Barts Experimental Cancer Medicine Centre, Barts Cancer Institute, Queen Mary University of London, London, United Kingdom

${ }^{2}$ Histogenex X N V, B 2610 Wilrijk, Belgium

${ }^{3}$ Hospital del Mar, Barcelona, Spain

${ }^{4}$ Instituto de Biomedicina de Sevilla, IBiS/Hospital Universitario Virgen del Rocio/CSIC/Universidad de Sevilla, Seville, Spain

${ }^{5}$ Southampton Experimental Cancer Medicine Centre, University of Southampton, Southampton, United Kingdom ${ }^{6}$ Department of Medical Oncology, The Netherlands Cancer Institute, Amsterdam, Netherlands 7 Institut Catala d'Oncologia, Hospital Universitari Germans Trias i Pujol, Badalona, Spain 8Institut Paoli-Calmettes, Marseille, France

${ }^{9}$ Hospital Clinico Universitario de Santiago, Santiago De Compostela, Spain

${ }^{10}$ Churchill Hospital, Oxford, United Kingdom

${ }^{11}$ Department of Medical Oncology, Hopital Saint-Andre, University of Bordeaux-CHU, Bordeaux, France

${ }^{12}$ Hospital Lyon SUD, Lyon, France

${ }^{13}$ Reina Sofia University Hospital, Cordoba, Spain

${ }^{14}$ Vall d'Hebron Institute of Oncology, Vall d'Hebron University Hospital, Universitat Autonoma de Barcelona, Barcelona, Spain

${ }_{15}$ UCLH, United Kingdom

${ }^{16}$ Medical Biochemistry, Faculty of Pharmaceutical, Biomedical and Veterinary Sciences, University of Antwerp, Belgium.

${ }^{17}$ Genentech, San Francisco USA.

${ }^{18}$ Hospital 12 de Octubre, Madrid, Spain 


\section{Introductory paragraph (225 words):}

Antibodies targeting programmed death 1 (PD-1) or the ligand 1 (PD-L1, such as atezolizumab) have significant efficacy in a proportion of metastatic urothelial cancers (UC) ${ }^{1,2}$. Biomarkers may facilitate identification of these responding tumors ${ }^{3}$. Neoadjuvant use of these agents is associated with pathological complete responses $(\mathrm{pCR})$ in a spectrum of tumors including $U \mathrm{C}^{4-7}$. Sequential tissue from these studies allows for detailed on treatment biomarker analysis. Here, we present a single arm phase II study, investigating two cycles of atezolizumab prior to cystectomy in 95 patients with muscle invasive UC (NCT02662309). pCR was the primary endpoint. Secondary endpoints focused on safety, relapse free survival and biomarker analysis. The pCR rate was $31 \%[95 \% \mathrm{Cl}: 21-41]$ achieving the primary efficacy endpoint. Baseline biomarkers showed pre-existing activated T cell expression was more prominent than expected and correlated with outcome. Other established biomarkers such as tumor mutational burden (TMB) did not predict outcome, differentiating this from the metastatic setting. Dynamic changes to gene expression signatures and protein biomarkers occurred with therapy, while changes in DNA alterations with treatment were uncommon. Responding tumors showed a predominance of expression of tissue repair genes after treatment making tumor biomarker interpretation challenging in this group. Stromal factors such transforming growth factor $\beta$ (TGF $\beta$ ) and fibroblast activation protein (FAP) were linked with resistance, as was high cell cycle gene signatures after treatment.

Funding: CRUK, UK Experimental Cancer Medicine Network and La Roche-Hoffmann Ltd. 


\section{Introduction (main text word count 2256).}

Cystectomy is the standard of care for patients with muscle invasive UC of the bladder who are not eligible for neoadjuvant chemotherapy ${ }^{8}$. Outcomes are poor and new treatments are required in this group of patients ${ }^{9,10}$. We investigated this population in a phase II study to establish efficacy, safety and biomarker signals. The neoadjuvant setting enabled sequential tissue sampling and the identification of molecular pathways associated with response and relapse. Three biologies described in the metastatic setting were initially investigated ${ }^{3}$ : (i) Pre-existing CD8+ T-cell immunity; (ii) transforming growth factor $\beta$ (TGF $\beta$ ), specifically in tumors with excluded immune phenotype; (iii) tumor mutation burden (TMB) in association with DNA damage repair (DDR) and cell cycle transcriptional signatures. Exploratory analysis of DNA alterations was also performed to identify novel makers of response and resistance.

\section{Results}

Between May 2016 and June 2018, 95 patients were recruited from 21 sites (Table 1). The median follow-up was 13.1 months [ $95 \% \mathrm{Cl}: 9.5$ - 13.5]. Eighty-eight patients were assessable for the primary endpoint (87 patients had cystectomy). Eight patients did not have cystectomy (3 treatment related) (Extended Data Figure 1). At the time of analysis 17 patients had relapsed and 17 patients had died (1 post-operative death, 1 treatment related death). The median time from starting atezolizumab to surgery was 5.6 weeks (IQR:4- 6.9).

The study met its primary endpoint with a pathological complete response $(p C R)$ rate of $31 \%(27 / 88)$ [95\% Cl: $21-41]$. pCR rates in patients previously treated with BCG $(n=10)$ were $30 \%$ [95\% Cl: $7-65]$, while the pCR rate in T3 or T4 disease at baseline was 17\% [95\% Cl: 5 - 37] (Extended Data Figure 2). One-year relapse free survival was $79 \%$ [95\% Cl: 67 - 87]. Radiological responses (RECIST v1.1) or progression prior to surgery occurred in $22 \%$ [95\% Cl: $13-35]$ and $16 \%$ [95\% Cl: $7-27$ ] respectively (Extended Data Figure 3).

No new safety signals were seen and treatment did not appear to complicate surgery, 39/87 (45\%) of patients had grade I to II surgical complication (Clavien Dindo classification) including urinary tract infection (26\%), paralytic ileus (7\%) and anaemia (6\%). 17\% patients had grade III to IV surgical complications, most commonly wound dehiscence (6\%). One patient had surgical complications resulting 
in post-operative death. Grade 3 or 4 CTC adverse reactions occurred in 10/95 (11\%) patients Extended Data Table 1. AEs that prevented cystectomy $(n=3)$ included deterioration of performance status, myocardial infarction and pneumonia.

Seven tumors exhibited characteristics of significant but incomplete response with immune infiltration and limited numbers of cancer cells remaining $(<10 \%)$. Such tumors have been characterized as major pathological response (MPR) in lung cancer and melanoma but have not been previously described in UC ${ }^{11,12}$ (Figure 1a). We defined them by the presence of $>90 \%$ necrosis, CD $8+T$ cell, macrophage and tertiary lymphoid follicles at the central histology review. This biological endpoint is considered to be important enough in lung cancer to be the primary endpoint in a randomised trial (NCT03800134). Further work in bladder cancer is required.

Overall, 35/88 (40\%) of patients were PD-L1 positive at baseline, which is higher than expected when compared to the metastatic UC population $(234 / 931,25 \%)^{2}$, tested with the same PD-L1 antibody clone (SP142 in immune cells $\geq 5 \%$ ). The pCR rate in this population was $37 \%$ [95\% Cl: $21-55]$ (Figure 1b), and the one-year relapse-free survival rate was $75 \%$ [95\% Cl: 53 - 87]. There was no correlation between PD-L1 expression and outcome, either on immune cells (Figure 1b) or tumor cells ( $p>0.05$ for both) (Extended Data Figure 4).

Pre-existing T cell immunity correlated with response. High intra-epithelial CD8+ expression was associated with a pCR rate of $40 \%$ [95\% Cl: 26 - 57] compared to $20 \%$ [95\% Cl: 9 - 35] with CD8$(p<0.05)$ (Figure 1b). The one-year relapse free survival was $85 \%$ [95\% Cl: $67-94]$ for the CD8+ population. A pre-defined 8-gene cytotoxic T-cell transcriptional signature 8 (TGE8) was significantly increased in responders at baseline compared to SD $(p<0.01)$ and relapse patients $(p<0.01)^{3}$ (Figure 1c).

Tumor-immune phenotypes characterised as CD8-inflamed, CD8-excluded and CD8-desert tumors have been previously described in metastatic UC cancer ${ }^{3}$. Excluded tumors are characterized by prominent stromal components, including fibroblasts and collagen ${ }^{3,13}$. Inflamed and desert phenotypes have been linked with response and resistance to atezolizumab respectively. We observed a higher than expected 
occurrence of $T$ - cell inflamed tumors compared to the metastatic setting $\left(73 \%\right.$ vs $\left.26 \%{ }^{3}\right)$ (Figure 1d). Inflamed immune phenotypes did not correlate with response due largely to its high prevalence across all phenotypes. We therefore explored the quality of the immune infiltrate with dual CD8 / Granzyme B (GZMB) staining. GZMB is an essential mediator in lymphocyte activity and is used as a surrogate marker of activated CD8 cells ${ }^{14}$. We showed CD8/GZMB dual stained cells were expressed in responding CD8 inflamed tumours phenotypes (14/16, 87\%), unlike in relapsing CD8-inflamed tumors, which showed low levels of CD8/GZMB+ve cells $(3 / 10,30 \%)(p<0.05)$ (Figure 1d). Therefore, the quality of the immune infiltrate is important in determining outcome.

Previous work in metastatic UC demonstrated that TGF $\beta$ drives resistance in the excluded immune phenotype by active $\mathrm{T}$ cell exclusion ${ }^{3}$. We reproduced these results in the neoadjuvant setting, broadening the relevance of the work (Figure 1e). The desert immune phenotype was not associated with response in this setting, alternative treatment approaches should be pursued. ${ }^{3}$

We next explored tumor intrinsic factors such as TMB, DNA damage repair (DDR) and cell cycle genes $^{15}$. The baseline median TMB was 10.09 mut./Mb [95\% Cl: $\left.3.78-42.87\right]$ (similar to the metastatic setting at 9.65 mut./Mb ${ }^{2}$ ) (Figure 1g). pCR rates were not increased in TMB high ( $\geq 10$ mut./Mb) (31\%) tumors (Figure 1h). Cell-cycle-regulation pathway genes ${ }^{3}$ and mutations in DDR genes also failed to correlate with outcome (Figure 1f and 1i and Extended Data Figure 5). Inconsistencies with the makeup of DDR signatures prohibit any cross-trial comparison. ${ }^{16}$

We next explored the effect of treatment on biomarker expression and observed a significant increase in intraepithelial CD8 (78\% increase in median values) (Figures 2a-b). Treatment was also associated with an increase in PD-L1, fibroblast activation protein (FAP) and CD8-GZMB+ expression $(p<0.05$ for each; Figure 2b). Positive pairwise correlation between these biomarkers were observed, except for the FAP/CD8 pairing (Extended Data Figure 6).

Changes in IHC biomarkers were then correlated with outcome. An increase in intraepithelial CD8 levels occurred in responding tumors, which was not the case in relapsing tumors $(p<0.01)$. FAP expression remained high in relapsing tumors while a decrease was seen in responders $(p<0.01)$ (Figure 2c). FAP 
plays an important role in immune resistance in the tumor microenvironment by recruiting inhibitory immune populations such as Tregs and subsets of myeloid cells ${ }^{17,18}$. FAP is a surrogate marker for cancer associated fibroblasts, which are present in the tumor microenvironment and associated with TGF $\beta$ and poor outcome ${ }^{17}$. FAP expression was linked with resistance to therapy, highlighting the importance of stromal components in determining outcome. This, along with the GZMB data, may explain why many inflamed tumors do not respond to therapy.

Dynamic changes to immune phenotypes occurred with therapy in 9/50 (18\%) patients with treatment (5 from excluded to inflamed and 4 from inflamed to excluded) (Extended Data Figure 7). The excluded immune phenotype was rare in relapsed patients $(n=2)$, limiting our ability to assess the relevance of stromal TGF $\beta$ signals in non-responder groups after treatment.

Molecular groups defined by the Lund classification have been previously defined and correlated with atezolizumab outcome in metastatic UC ${ }^{19}$. In the neoadjuvant setting, we found that most tumors $(41 / 64$, 64.1\%) change taxonomy group with therapy (Figure $\mathbf{2 d}$ ). Following treatment, most responsive tumors $(14 / 15,93.3 \%)$ were classified as "infiltrated" , with increased immune infiltrate, angiogenesis and stromal gene signatures, and decreased cell cycle and DDR signatures. Further gene expression analysis in responding tumors ( $\mathrm{PCR}$ and MPR) showed similar patterns with high expression of the extra cellular matrix and collagen formation (Figure 2e). Therefore, the post-treatment transcriptome of responding tumors reflects tumor micro-environment re-organisation with reduced tumor related genes. While previous works demonstrated intratumoral heterogeneity for TCGA subtypes, these data show consistent changes in responding tumors focusing on host dominated tissue repair ${ }^{20}$. This has implications for future research, in that, responding samples after treatment appear to reflect host rather than tumor tissue. Due to these limitations, we compared the transcriptional and DNA profiles of stable disease and relapsed tumors in the treated samples (excluding responding patients). Treatment did not have a significant effect on TMB suggesting that the two-cycle treatment course is too short to promote expansion of resistant somatically-mutated tumor clones. TMB or DDR alterations did not predict relapse in treated samples (Figure 2f). However, proliferation/cell cycle gene expression was associated with relapse in treated samples $(p=0.02)$ (Figures $\mathbf{2 g}-\mathbf{h})$. Speculation surrounding a link between cell cycle/proliferation genes and outcome has been proposed in glioblastoma ${ }^{6}$. It is an avenue for further 
research and novel therapeutic combinations in the future (NCT01676753). Up regulation of cell cycle genes could represent more aggressive tumor phenotypes. Alternatively, preclinical data suggests cell cycle gene over expression may enhance resistant immune cell expression, highlighting a possible mechanism of immune escape ${ }^{21}$.

Exploratory mutation analysis identified increased amplification of FGF3, FGF19 and CCND1, which are on the same locus (Ch11q13.3), in responding tumors (Figure 3a). Drugs targeting FGF have recently been approved in UC by the FDA ${ }^{22}$. This association between FGF alterations and response has not been well described, indeed it contradicts previous speculation from TCGA analysis ${ }^{13}$. Combination trials targeting PD-L1 and cell cycle genes are justified. Consistent DNA alterations were not observed between pre- and post-treatment samples, suggesting stability rather than rapid clonal evolution during this short period on therapy (Figure 3b). This is consistent with our TMB data. In view of this, treated tissue was not correlated with outcome. Previous data in the neoadjuvant area is inconsistent here. This may be driven by relatively small numbers in previous studies, different agents or duration of therapy ${ }^{5,12}$. Finally, we compared gene expression before and after treatment in tumors which were stable with therapy. The stable disease samples were selected to minimise the effect of the host response on expression as seen in the responding patients. (Figures $\mathbf{3 c - d}$ ). Results showed increased immune signatures associated with therapy, consistent with the mechanism of action of therapy.

Previous studies in the metastatic setting have attempted to combine biomarker to improve patients selection, e.g. high TMB and highTeffector ${ }^{3,13,15}$. We performed exploratory analysis with combinations of biomarker in an attempt to enrich for responses. Specifically we investigated tGE8 signature described previously in figure 1c with either TMB or CD8/GZMB (Figure 3e; Extended data 8). Results showed the dual expression of CD8/GZMB and tGE8 $(n=25)$ was associated with a $40 \%$ response rate and $4 \%$ relapse rate. No enrichment was seen in the TMB/tGE8 combination.

Our trial reported biomarker analysis on over 60 paired samples with outcome data, which is more robust that previous neoadjuvant series across four tumor types ${ }^{5-7,11,12}$. Tissue in this study was taken at a specific time point and treatment was given for a specific duration, which may influence results. Our work highlights the difficultly in response biomarkers after treatment due to the limitation of cancer tissue. This 
requires consideration in future studies. A high proportion of patients had node positive disease and relapse rate at 1 year was $21 \%$, suggesting adjuvant therapy is required to maximise outcomes. Results from 1- year adjuvant atezolizumab are awaited (NCT03024996).

\section{Discussion}

There are currently no peri-operative systemic treatments recommended for operable muscle invasive UC not eligible for neoadjuvant chemotherapy, despite high cancer related mortality. Therefore, the significant activity shown here with atezolizumab is clinically relevant. Validation of $\mathrm{pCR}$ and MPR as a surrogate marker of outcome for immune checkpoint inhibitors is required. In our study only 2 of 29 patients with $\mathrm{pCR}$ or MPR have relapsed.

Biomarker results from tissue taken prior to starting therapy showed two of the three previously identified pathways associated with outcome remain relevant ${ }^{3}$. Higher than expected pre-existing $\mathrm{T}$ cell immunity appears to be the driving factor accounting for the higher than expected response rates. This appears a major discriminating feature between this and the metastatic setting ${ }^{1-3}$. The quality of immune infiltration is relevant in generating response, as highlighted by the CD8-GZMB+ve T cells data. The failure of TMB and DDR signatures to correlate with outcome is a second discriminating factor from the metastatic setting. This was seen despite comparable median TMB levels in the metastatic and perioperative setting (9.6 vs 10.1 Mut./MB respectively) ${ }^{2}$. Correlation of TMB with outcome in the neoadjuvant setting has been inconsistent in other smaller neoadjuvant trials ${ }^{5,7}$. These neoadjuvant studies employed different drugs for different durations across a spectrum of tumor types which may partly at least account for the inconsistencies. T cell biomarkers to predict response may be preferable to existing biomarkers (PD-1), especially in combination, and require testing in ongoing randomised trials (NCT03732677).

Our findings directly contrast those seen in metastatic, chemotherapy resistant UC with atezolizumab 2,13,15. This reversal of biomarker relevance implies that their performance with anti-PD-L1 treatment is dependent on the clinical setting in UC. TCGA data, our gene expression data, and DNA analysis demonstrate changes in the biology of UC with more advanced disease ${ }^{16,23}$. We speculate that different treatment and biomarker approaches will be required to maximise outcomes in different clinical setting. 
The biomarkers identified here, especially from post treatment tissue may facilitate patient selection and gives a better understanding of the biology of the disease.

\section{Methods}

Trial design and patient population: This is an open-label, international, multicentre, single arm, neoadjuvant phase II trial evaluating the effects of two cycles (1200mg, 3 weekly) of preoperative atezolizumab in patients with histologically confirmed (T2-T4aNOMO) transitional cell UC of the bladder, awaiting planned radical cystectomy. Additional eligibility criteria included residual disease after transurethral resection of the bladder (TURBT), adequate fitness for planned cystectomy (according to local guidelines), ineligible for or refusal of cisplatin based neoadjuvant chemotherapy, no evidence of nodal or metastatic disease on cross sectional imaging, Eastern Cooperative Oncology Group (ECOG) Performance Status of 0 or 1 and adequate hematologic and end-organ function within 4 weeks prior to the first study treatment. Major exclusion criteria included evidence of significant uncontrolled concomitant disease that could affect compliance with the protocol or interpretation of results, previous autoimmune disease, ongoing active infections or prior use of immune checkpoint inhibitors. All patients provided written informed consent. The relevant institutional review board or ethics committee for each participating centre approved the study, which was conducted in accordance with the principles of Good Clinical Practice, the provisions of the Declaration of Helsinki, and other applicable local regulations. The study was sponsored by Queen Mary University of London (NCT02662309). The Barts Experimental Cancer Centre Clinical (ECMC) Trials Group had overall responsibility for trial management; the trial management group was responsible for day-to-day running of the trial and the trial was overseen by an independent data monitoring committee (IDMC). Emerging safety data was reviewed regularly by the IDMC.

Clinical endpoints and statistical considerations: Pathological complete response $(p C R)$ rate (investigator assessed) was selected as the primary efficacy endpoint. Efficacy analysis for the pCR rate included all patients who met the eligibility criteria, had at least one cycle of atezolizumab, and underwent cystectomy or withdrew for progression of disease. pCR was defined as pT0 and in situ cancer (TIS) based on histological evaluation of the TURBT and cystectomy samples by local institutional analysis. A pCR of $\geq 20 \%$ would support further investigation and the pCR should not be $10 \%$ 
or less. A'Hern's single stage design ${ }^{24}$ was used to yield a sample size of 78 patients $(80 \%$ power and one-sided test of significance at the $5 \%$ level). To allow for withdrawal of consent and possible drop-outs or patients who did not undergo cystectomy the intention was to recruit approximately 85 assessable patients. Sample size calculations were performed using the software package PASS version 12.0

Secondary endpoints included PCR rate in PD-L1 positive patients. The standard definition of PD-L1 positivity for atezolizumab in bladder cancer was used ( $\geq 5 \%$ of immune cells staining using the SP142 antibody, Ventana11). Response rate (RR) was evaluated on cross-sectional imaging by RECIST v1.1. Adverse Events (AEs) graded according to NCI-CTCAE v4.03 were collected during treatment and up to 24 weeks post-cystectomy. Surgical complications were assessed using the Clavien Dindo classification up to 4 weeks post-cystectomy. Safety analysis, RR and relapse free survival (RFS) are presented for all patients who met the eligibility criteria and received study drug. RFS is a discriminatory endpoint and the primary endpoint of most of the randomised trials. pCR remains attractive in the short term but has not been validated with immune oncology drugs in urothelial cancer.

All clinical efficacy endpoints were analysed using STATA version 13.1. The Kaplan-Meier method was used to measure time to disease recurrence. Relapsed definition included radiological or clinical relapse. Wilcoxon rank sum test was used to compare changes in biomarkers before and after therapy. Correlations were measured by Pearson product moment correlation coefficient. All IHC protein tests were performed in $\mathrm{R}$.

Central pathology review and protein analysis: A central pathology review of PanCK-CD8 and PD-L1 occurred on all patients who had available tumor tissue at baseline (92) and cystectomy (84) respectively (Extended Data Figure 1). Not all patients had all pre/ post biomarkers measured due to lack of tumor tissue availability in PCR samples. PD-L1 and PanCK-CD8 were initially prioritized. Immune phenotypes (inflamed, desert, excluded) samples were assessed by two trained histopathologists in 78 baseline and 57 post-treatment (scored on the PanCK-CD8)9. PD-L1 (SP142), PanCK (AE1/AE3/PCK26), CD8 (SP239), GZMB (EPR8260) and FAP (SP325) antibodies were used for biomarker analysis using established methods on the Ventana BenchmarkR ULTRA and Ventana BenchmarkR XT platform. PanCK-CD8, CD8-GZMB and FAP were scored via a quantitative method using the image analysis 
software VisiopharmR in the total tumor area. Low, medium and high FAP expression was measured in the tumor stroma area. PD-L1, PanCKCD8, FAP and CD8-GZMB levels above and below the median were compared. In the PanCKCD8 analysis, the values of CD8 cells within the cytokeratin-positive tumor strands were used. Major pathological responses with immune checkpoint inhibitors have been described previously ${ }^{11}$. Features included immune / macrophage infiltration, tumor necrosis and neovascularisation. The characteristics of MPR in muscle invasive UC of the bladder were assessed in this study.

RNA sample procurement and processing: FFPE tissue was macro-dissected for tumor area using H\&E as a guide. RNA was extracted using the High Pure FFPET RNA Isolation Kit (Roche) and assessed by Qubit and Agilent Bioanalyzer for quantity and quality. First strand cDNA synthesis was primed from total RNA using random primers, followed by the generation of second strand cDNA with dUTP in place of dTTP in the master mix to facilitate preservation of strand information. Libraries were enriched for the mRNA fraction by positive selection using a cocktail of biotinylated oligos corresponding to coding regions of the genome. Libraries were sequenced using the Illumina sequencing-bysynthesis.

RNA-seq data generation and processing: Raw RNA-seq counts were obtained from Genentech's internal stranded count pipeline. Raw counts were adjusted for gene length using transcript-per-million (TPM) normalization, and subsequent log2-transformation.

DNA sample procurement: FFPE tissue was macro-dissected for tumor area using H\&E as a guide. DNA was extracted using KingFisher (Thermo Scientific) and analyzed at Foundation Medicine using the FoundationOneCDx assay. Genomic analyses including TMB were performed by Foundation Medicine.

DNA somatic mutation data: Comprehensive genomic profiling (CGP) was carried out in a Clinical Laboratory Improvement Amendments (CLIA)-certified, College of American Pathologists (CAP)accredited laboratory (Foundation Medicine Inc., Cambridge, MA, USA) on all-comers during the course of routine clinical care. Approval was obtained from the Western Institutional Review Board (Protocol No. 20152817). Hybrid capture was carried out for all coding exons from up to 395 cancer-related genes plus select introns from up to 31 genes frequently rearranged in cancer. We assessed all classes of genomic 
alterations (GA) including short variant, copy number, and rearrangement alterations, as described previously. Biallelic $(\mathrm{CN}=0)$ copy number loss was called as previously described. Shallow copy-number loss $(\mathrm{CN}=1)$ was called using similar methodology to arm-level calling. Normalized coverage data for exonic, intronic, and SNP targets accounting for stromal admixture were plotted on a logarithmic scale and minor allele SNP frequencies were concordantly plotted. Custom circular binary segmentation further clustered targets and minor allele SNPs to define upper and lower bounds of genomic segments. Signal-to-noise ratios for each segment were used to determine whether it was gained or lost. The sum of those segment sizes determined the fraction of each segment gained or lost.

Statistical methods: Unless otherwise stated, all two-group comparisons for continuous variables use the two-sided Mann-Whitney test (R function, Wilcoxon test). For categorical variables, the Pearson's Chi-squared test with continuity correction is used. Unless otherwise stated, FDR corrected p-values are reported. Measurements were taken from distinct samples.

Data availability: Data that support the findings of this manuscript are available from the corresponding author upon reasonable request. All raw sequencing data required for RNA-seq analyses will be deposited to the European Genome-Phenome Archive. 


\section{Conflicts of interest}

Employment by Roche: SM, JT, RB; Employment by Histogenex: MK, PJ, SD; Research funding/Honoraria BMS: TP, AR, ID, MV, PA, ML; Research funding/Honoraria Roche: TP, AR, ID, MV, GG, UA, MK, PA, AR, MJM, CS; Research funding/Honoraria AZ: TP, ID, MV, AP, GG, UA, MK, CS; Research funding/Honoraria MSD: TP, ID, SC, MV, MK, DM, MJM, CS, ML; Research funding/Honoraria Pfizer: TP, SC, GG, PA, AR, MJM, CS; Research funding/Honoraria Novartis: ID, UA, PA, AR, CS; Research funding/Honoraria Exelixis: TP, CS; Research funding/Honoraria Astellas: TP, SC, GG, PA, AR, ML; Research funding/Honoraria Johnson and Johnson: TP.

Thomas Powles (TP), Mark Kockx (MK), Alejo Rodriguez-Vida(AR), Ignacio; Duran (ID), Simon J. Crabb (SC), Michiel S. Van Der Heijden (MV), Bernadett Szabados(SB), Albert Font Pous (AP), Gwenaelle Gravis(GG), Urbano Anido Herranz (UA), Andrew Protheroe (PA), Alain Ravaud (AR), Denis Maillet (DM), Maria Jose Mendez (MJM), Cristina Suarez(CS), Mark Linch (ML), Aaron Prendergast (AP), Pieter-Jan van Dam (PJ), Diana Stanoeva,(DS) Sofie Daelemans(SD), Sanjeev Mariathasan(SM), Joy Tea(JT), Kelly Mousa (KM), Romain Banchereau (RM), Daniel Castellano (DC). 


\section{References}

1. Bellmunt, J., Powles, T. \& Vogelzang, N.J. A review on the evolution of PD-1/PD-L1 immunotherapy for bladder cancer: The future is now. Cancer Treat Rev 54, 58-67 (2017).

2. Powles, T., et al. Atezolizumab versus chemotherapy in patients with platinum-treated locally advanced or metastatic urothelial carcinoma (IMvigor211): a multicentre, open-label, phase 3 randomised controlled trial. Lancet 391, 748-757 (2018).

3. Mariathasan, S., et al. TGFbeta attenuates tumour response to PD-L1 blockade by contributing to exclusion of T cells. Nature 554, 544-548 (2018).

4. Blank, C.U., et al. Neoadjuvant versus adjuvant ipilimumab plus nivolumab in macroscopic stage III melanoma. Nat Med 24, 1655-1661 (2018).

5. Necchi, A., et al. Pembrolizumab as Neoadjuvant Therapy Before Radical Cystectomy in Patients With Muscle-Invasive Urothelial Bladder Carcinoma (PURE-01): An Open-Label, Single-Arm, Phase II Study. J Clin Oncol, JCO1801148 (2018).

6. Cloughesy, T.F., et al. Neoadjuvant anti-PD-1 immunotherapy promotes a survival benefit with intratumoral and systemic immune responses in recurrent glioblastoma. Nat Med 25, 477-486 (2019).

7. Amaria, R.N., et al. Neoadjuvant immune checkpoint blockade in high-risk resectable melanoma. Nat Med 24, 1649-1654 (2018).

8. Reardon, Z.D., et al. Trends in the use of perioperative chemotherapy for localized and locally advanced muscle-invasive bladder cancer: a sign of changing tides. Eur Urol 67, 165-170 (2015).

9. International Collaboration of, T., et al. International phase III trial assessing neoadjuvant cisplatin, methotrexate, and vinblastine chemotherapy for muscle-invasive bladder cancer: long-term results of the BA06 30894 trial. J Clin Oncol 29, 2171-2177 (2011).

10. Kim, H.S., Jeong, C.W., Kwak, C., Kim, H.H. \& Ku, J.H. Pathological T0 Following Cisplatin-Based Neoadjuvant Chemotherapy for Muscle-Invasive Bladder Cancer: A Network Meta-analysis. Clin Cancer Res 22, 1086-1094 (2016).

11. Forde, P.M., et al. Neoadjuvant PD-1 Blockade in Resectable Lung Cancer. N Engl J Med 378, 1976-1986 (2018).

12. Huang, A.C., et al. A single dose of neoadjuvant PD-1 blockade predicts clinical outcomes in resectable melanoma. Nat Med 25, 454-461 (2019). 
13. Rosenberg, J.E., et al. Atezolizumab in patients with locally advanced and metastatic urothelial carcinoma who have progressed following treatment with platinum-based chemotherapy: a singlearm, multicentre, phase 2 trial. Lancet 387, 1909-1920 (2016).

14. Nowacki, T.M., et al. Granzyme B production distinguishes recently activated CD8(+) memory cells from resting memory cells. Cell Immunol 247, 36-48 (2007).

15. Powles, T., et al. Atezolizumab (atezo) vs. chemotherapy (chemo) in platinum-treated locally advanced or metastatic urothelial carcinoma (mUC): Immune biomarkers, tumor mutational burden (TMB), and clinical outcomes from the phase III IMvigor211 study. Journal of Clinical Oncology 36, 409-409 (2018).

16. Teo, M.Y., et al. Alterations in DNA Damage Response and Repair Genes as Potential Marker of Clinical Benefit From PD-1/PD-L1 Blockade in Advanced Urothelial Cancers. J Clin Oncol 36, 1685-1694 (2018).

17. Wynn, T.A. \& Ramalingam, T.R. Mechanisms of fibrosis: therapeutic translation for fibrotic disease. Nat Med 18, 1028-1040 (2012).

18. Richards, K.E., et al. Cancer-associated fibroblast exosomes regulate survival and proliferation of pancreatic cancer cells. Oncogene 36, 1770-1778 (2017).

19. Sjodahl, G., et al. A molecular taxonomy for urothelial carcinoma. Clin Cancer Res 18, 3377-3386 (2012).

20. Warrick, J.I., et al. Intratumoral Heterogeneity of Bladder Cancer by Molecular Subtypes and Histologic Variants. Eur Urol 75, 18-22 (2019).

21. Sun, L., et al. WEE1 kinase inhibition reverses G2/M cell cycle checkpoint activation to sensitize cancer cells to immunotherapy. Oncoimmunology 7, e1488359 (2018).

22. Bahleda, R., et al. Multicenter Phase I Study of Erdafitinib (JNJ-42756493), Oral Pan-Fibroblast Growth Factor Receptor Inhibitor, in Patients with Advanced or Refractory Solid Tumors. Clin Cancer Res (2019).

23. Nassar, A.H., et al. Mutational Analysis of 472 Urothelial Carcinoma Across Grades and Anatomic Sites. Clin Cancer Res 25, 2458-2470 (2019).

24. A'Hern, R.P. Sample size tables for exact single-stage phase II designs. Stat Med 20, 859-866 (2001). 


\begin{tabular}{|c|c|c|c|}
\hline & $\begin{array}{c}\text { Treated } \\
\text { population } \\
(\mathrm{N}=95)\end{array}$ & $\begin{array}{c}\text { Clinical primary } \\
\text { endpoint } \\
\text { population } \\
(\mathrm{N}=88)\end{array}$ & $\begin{array}{l}\text { PCR population } \\
(\mathrm{N}=27)\end{array}$ \\
\hline Age (years), median (IQR) & $\begin{array}{c}73 \\
(68-77)\end{array}$ & $\begin{array}{c}72 \\
(67-76)\end{array}$ & $\begin{array}{c}73 \\
(68-79)\end{array}$ \\
\hline Male gender, (\%) & $81(85)$ & $75(85)$ & $24(89)$ \\
\hline \multicolumn{4}{|l|}{ TNM stage, $\mathbf{n}(\%)$} \\
\hline T2 & $70(74)$ & $64(73)$ & $23(85)$ \\
\hline T3 & $17(18)$ & $17(19)$ & $3(11)$ \\
\hline T4 & $8(8)$ & $7(8)$ & $1(4)$ \\
\hline N positive & 0 & 0 & 0 \\
\hline M1 & 0 & 0 & 0 \\
\hline Previous non-muscle invasive disease, $n(\%)$ & $14(15)$ & $14(16)$ & $3(11)$ \\
\hline Tis & $1(1)$ & $1(1)$ & 0 \\
\hline Ta & $5(5)$ & $5(6)$ & 0 \\
\hline T1 & $8(8)$ & $8(9)$ & $3(11)$ \\
\hline CIS & $5(5)$ & $5(6)$ & $1(4)$ \\
\hline Previous BCG, n(\%) & $11(12)$ & $10(11)$ & $3(11)$ \\
\hline Current or previous smoker, $\mathrm{n}(\%)$ & $74(78)$ & $69(78)$ & $23(85)$ \\
\hline Radiological measurable disease, n(\%) & $69(73)$ & $65(74)$ & $16(59)$ \\
\hline PD-L1 positivity, n(\%) & $39(41)$ & $35(40)$ & $13(48)$ \\
\hline Median intratumoral CD8 count & $186 \cdot 25$ & 173.97 & $216 \cdot 25$ \\
\hline \multicolumn{4}{|l|}{ ECOG Performance status, $\mathrm{n}(\%)$} \\
\hline 0 & $71(75)$ & $70(80)$ & $24(89)$ \\
\hline 1 & $24(25)$ & $18(20)$ & $3(11)$ \\
\hline Haemoglobin, median (IQR) & $\begin{array}{c}133 \cdot 0 \\
(120 \cdot 0-143 \cdot 4)\end{array}$ & $\begin{array}{c}132 \cdot 5 \\
(121 \cdot 0-143 \cdot 2)\end{array}$ & $\begin{array}{c}133 \cdot 0 \\
(126 \cdot 0-146 \cdot 0)\end{array}$ \\
\hline Albumin, median (IQR) & $\begin{array}{c}43 \cdot 0 \\
(39.0-45 \cdot 6)\end{array}$ & $\begin{array}{c}43 \cdot 0 \\
(39.0-46 \cdot 0)\end{array}$ & $\begin{array}{c}44 \cdot 0 \\
(41 \cdot 0-46 \cdot 0)\end{array}$ \\
\hline eGFR $\leq 60 \mathrm{~mL} / \mathrm{min}, \mathrm{n}(\%)$ & $39(41)$ & $36(41)$ & $11(41)$ \\
\hline
\end{tabular}

Table 1 Patients characteristics at baseline. $B C G=$ Bacillus Calmette-Guérin. $C I S=$ Carcinoma in situ. $\mathrm{ECOG}=$ Eastern Cooperative Oncology Group. IQR = Interquartile range. All percentages are based on the total number of patients in the relevant population $(\mathrm{N})$. 


\section{Figure 1}

a
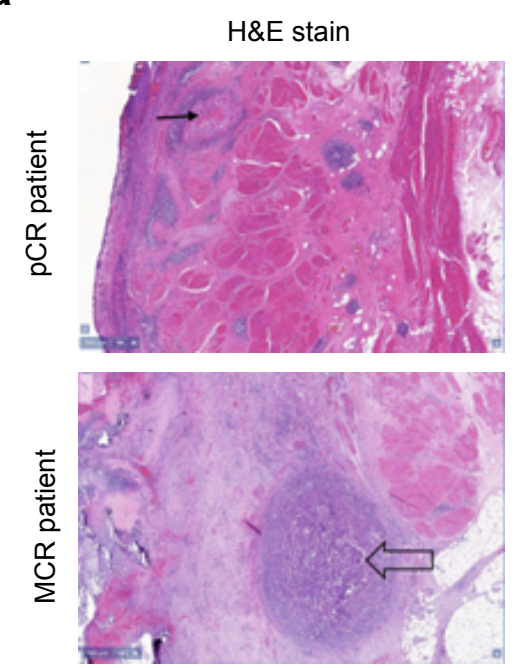

C

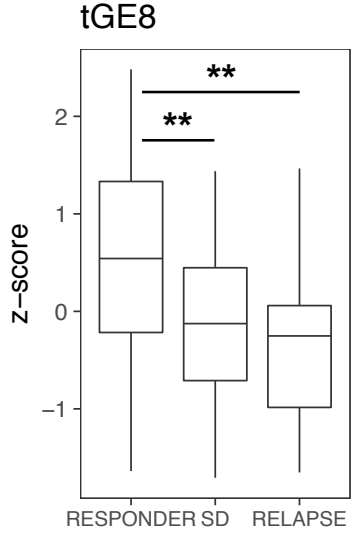

d

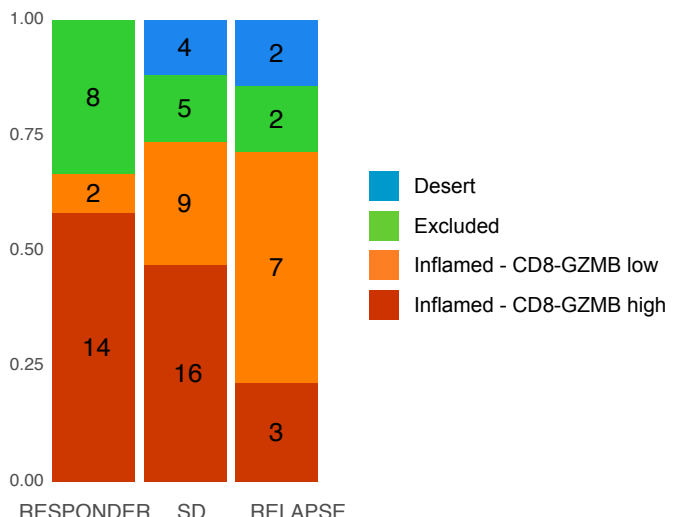

f

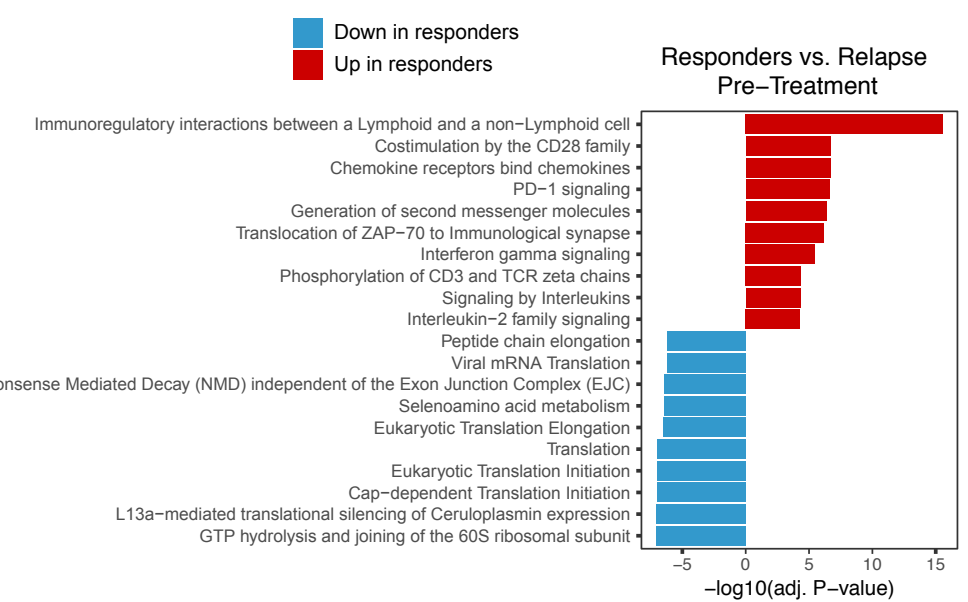

h
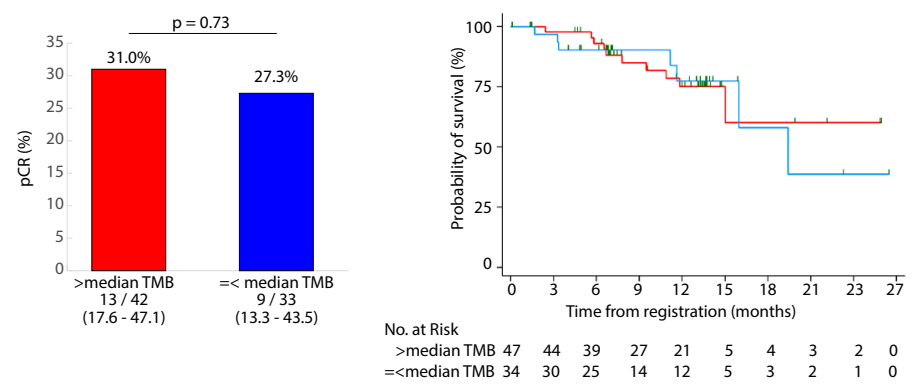

b
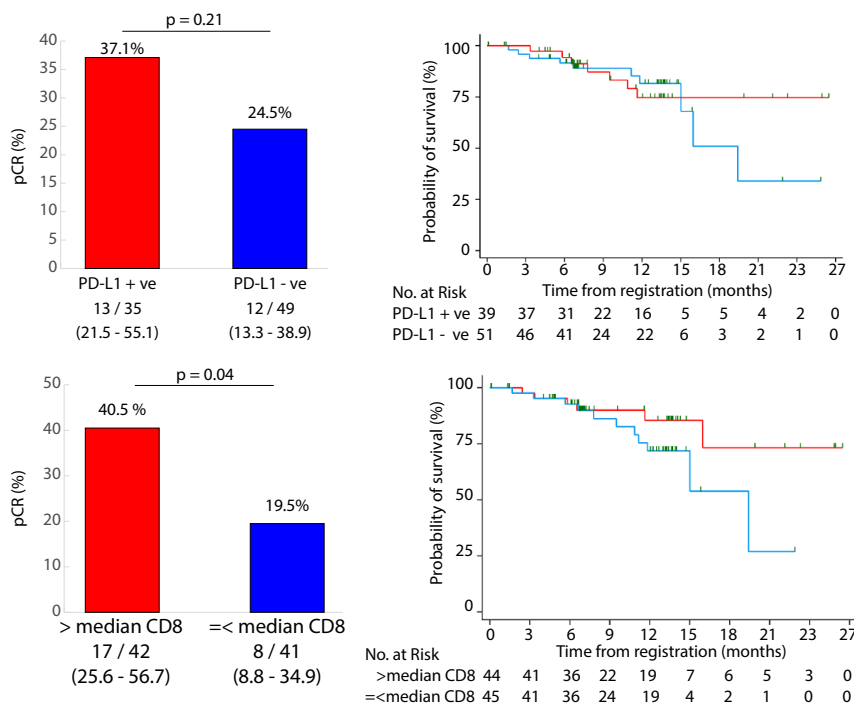

$\begin{array}{llllllllll}\text { No-L1 + ve } 39 & 37 & 31 & 22 & 16 & 5 & 5 & 4 & 2 & 0 \\ \text { PD-L1 - ve 51 } & 46 & 41 & 24 & 22 & 6 & 3 & 2 & 1 & 0\end{array}$

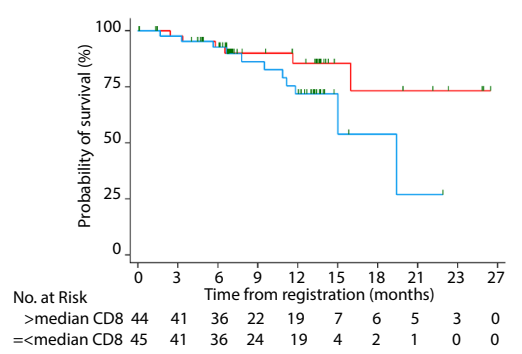

e

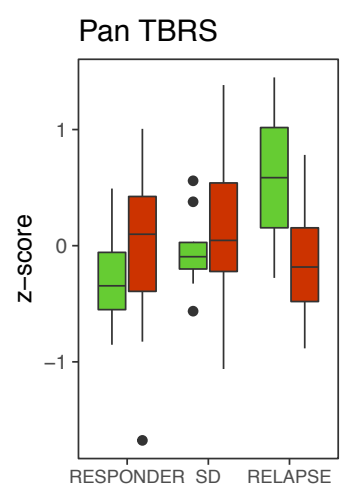

tGE8 / Pan TBRS

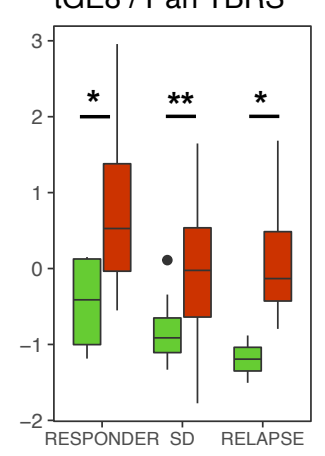

Immune Phenotype

Excluded Inflamed

g
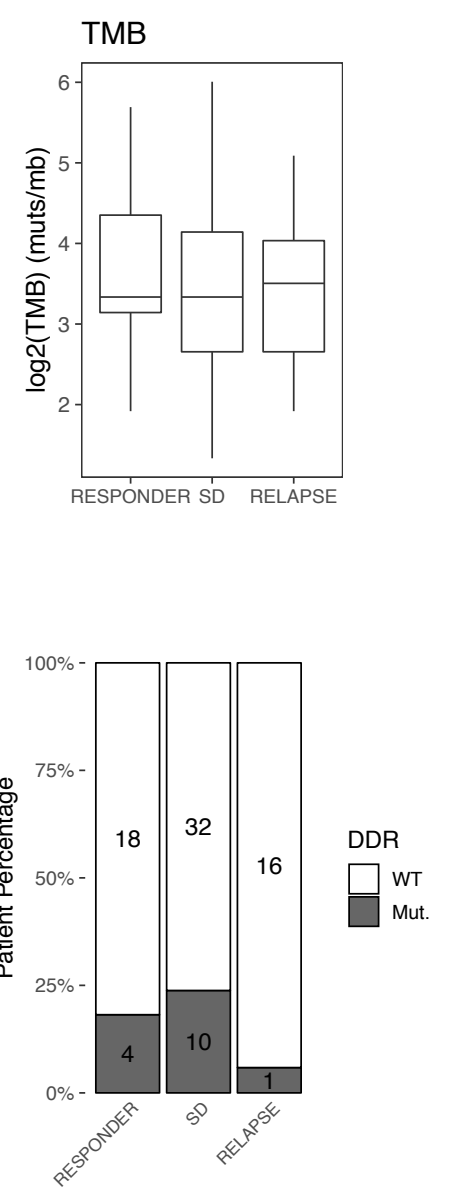
FIGURE 1: Analysis of three response biologies at baseline. Longitudinal tumor samples were obtained at baseline (TURBT) and after completion of 2 cycles of neoadjuvant treatment with atezolizumab and undergoing radical cystectomy. The tumor molecular and immune microenvironment was compared between responders (patients achieving PCR and MPR on histopathological review), stable disease and patients presenting relapsed or metastatic disease (assessed clinically or on subsequent imaging) on baseline tissue.

a: Representative images of cystectomy samples showing pathological complete response (pCR) and major pathological response (MPR) after neoadjuvant treatment. The first row shows pCR characterized by the absence of tumor cells and areas of necrosis surrounded by CD8 cells (arrowed), by H\&E (left) or PanCK-CD8 stain (right). The second row shows MPR defined as $10 \%$ or less of residual viable tumor cells of the removed primary tumor, dense CD8 and macrophage infiltration as well as numerous tertiary lymphoid follicles. Arrows indicate immune cells encircling residual tumor. The tumor bed region of the MPR patients was recognized by a anatomic pathologist in the paraffin blocks of the cystectomy specimen. For optimal evaluation the pathologist combined the H\&E with PanCytokeratin-CD8 slides. Residual tumor cell groups were recognized. Serial sections were IHC stained for the different proteins and scanned to generate whole slide images. This was followed by an alignment step, allowing the study of protein expression in the same regions of the major pathological response.

b: pCR percent (left panels) and time-to-relapse or cancer-related death (right panels) stratified by baseline PD- L1 status (upper panels), intraepithelial CD8 expression (lower panels) levels. PDL1: positive $=$ IC staining of $\geq 5 \%$ with SP142 antibody ${ }^{13}$; CD8: above vs. below median 186.25 . All patients were included in the relapse-free survival analysis (Kaplan Meier; RECISTv1.1). Not all patients had surgery for pCR assessment. This accounts for discrepancies between the two plots. One-year relapse- free survival for the PD-L1+ population and CD8high population was 75\% [95\% CI: 53 - 87], 85\% [95\% Cl: 67 - 94] respectively. Comparative statistical testing was not performed on relapse-free survival due to maturity of data. 
c: tGE8 gene signature expression by clinical outcome at baseline. tGE8 is a transcriptional signature of 8 genes (IFN CXCL9, CD8A, GZMA, GZMB, CXCL10, PRF1, TBX21) described previously in locally advanced or metastatic UC ${ }^{3}$, which represents IFN- signalling and CD8 effector T cell presence. The signature $z$-score is calculated as the median of the $z$-score for each gene from the signature. On box plots, the horizontal bold line represents the median. The lower and upper hinges of the box correspond to the first and third quartiles (the 25th and 75th percentiles). The upper whisker extends from the hinge to the largest value no further than $1.5^{\star}$ IQR from the hinge (where IQR is the inter-quartile range, or distance between the first and third quartiles). The lower whisker extends from the hinge to the smallest value at most 1.5

${ }^{*}$ IQR of the hinge. Points represent outliers. ${ }^{* *}$ represents $p$-value $<0.01$.

\section{d: Distribution of CD8 immune phenotypes by clinical outcome group at baseline. Immune} phenotypes were assessed centrally by two pathologists using established methods ${ }^{15} .78$ baseline samples were assessed. A high prevalence of inflamed phenotype baseline were observed in this cohort (inflamed $=73 \%$, excluded $=19 \%$, desert $=8 \%$ ). There was a predominance of inflamed tumors, unlike the metastatic setting ${ }^{2}$. No clear correlation between phenotype and response was apparent. Inflamed tumors were then split into two groups expressing high and low dual CD8/GZMB+ve staining. There is a significant correlation $(\mathrm{p}=0.004)$ between $C D 8 / G Z M B+v e$ dual stain cells and response in CD8-inflamed tumor phenotypes, unlike in relapsing tumors which showed low levels of CD8/GZMB +ve cells. This suggests the quality of the immune infiltrate is relevant in determining response.

\section{e: Association between clinical outcome and TGF $\beta$-induced genes (pan TBRS signature) at baseline by immune phenotype: The TBRS signature includes 19 genes induced by TGF $\beta$ in in vitro experiments ${ }^{3}$. At baseline, the Pan- TBRS signature exhibits a statistically non-significant trend for increase in excluded tumors from patients that will relapse $(n=2)$. These data support previous results in metastatic UC ${ }^{3}$ The panel on the right combines tGE8 and TBRS signature correlates with immune phenotype and response to treatment. * and ${ }^{* *}$ represents $p$-values ( 0.05 and 0.01 respectively).}


f: Reactome analysis comparing response and relapse tumors from baseline tissue. Top gene sets associated with response or relapse are displayed. Association with the cell cycle signature (MKI67, CCNE1, BUB1, BUB1B, CCNB2, CDC25C, CDK2, MCM4, MCM6, MCM2) was not significant ${ }^{15}$.

g: Relationship of TMB at baseline and the correlation with outcome. The total tumor mutational burden was estimated using the Foundation One method ${ }^{2}$ as previously described in UC. No correlation was observed between TMB and response to treatment.

h: pCR percent (left panels) and time-to-relapse or cancer-related death (right panels) stratified by TMB levels: above vs. below median (10.1m/MB). No significant correlation was seen. One-year relapse- free survival for the TMBhigh population was $75 \%$ [95\% $\mathrm{Cl}: 57$ - 86].

i: DDR alteration status by outcome. No significant difference $(p=0.28)$ in DDR mutation signature between outcome groups at baseline. Note that only 26 genes had detectable alterations in at least one sample. 


\section{Figure 2}

a
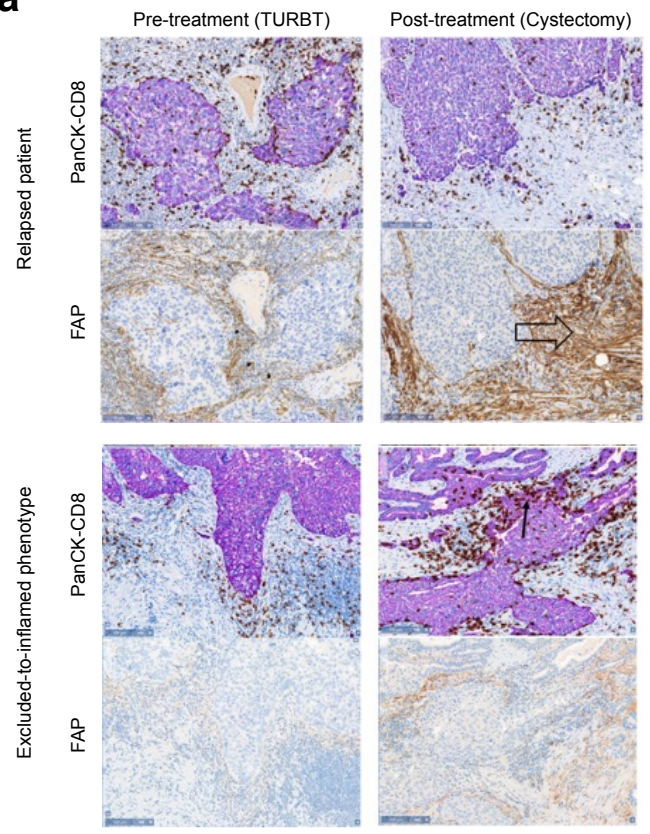

d

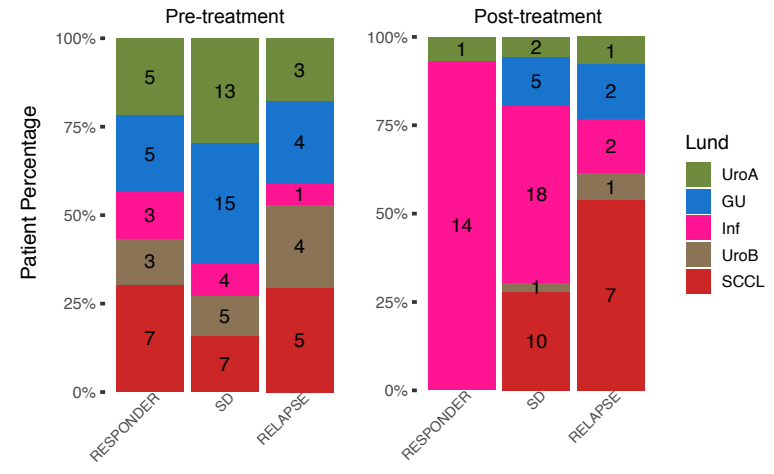

f

TMB

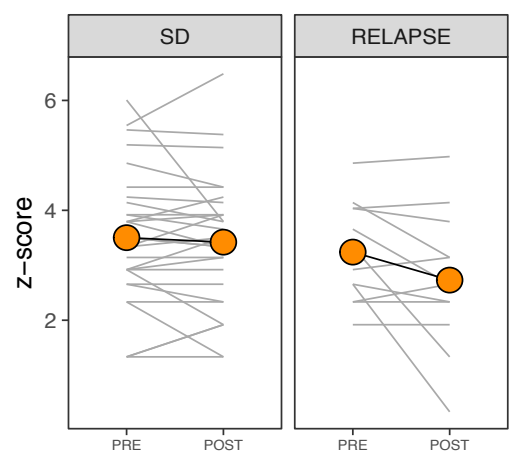

h

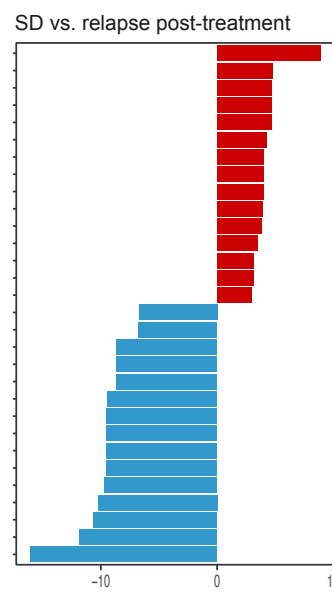

$-\log 10($ adj. P-value) b
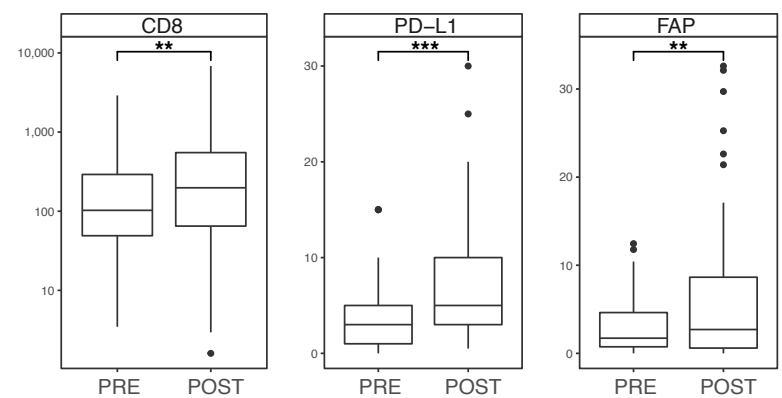

C
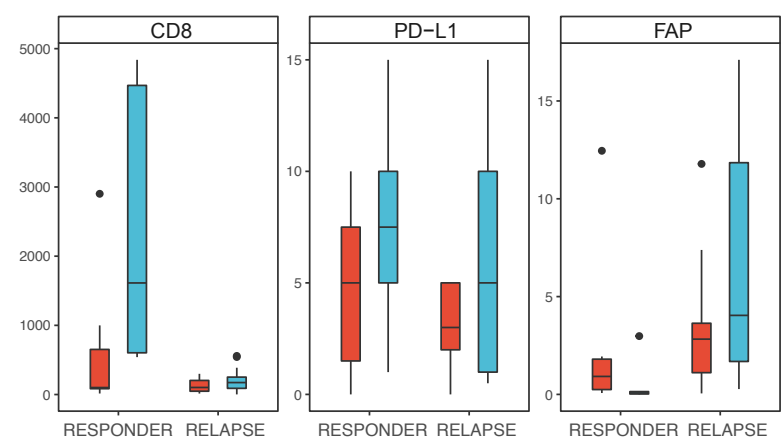

Visit

昂 PRE

e

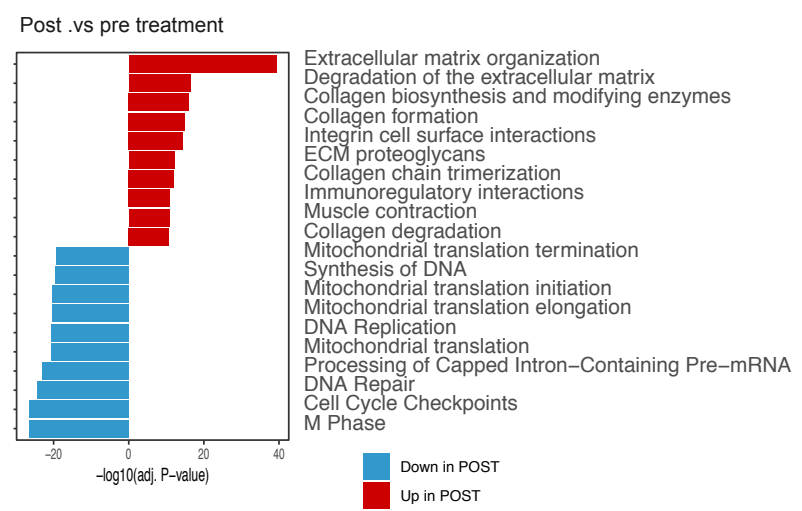

9

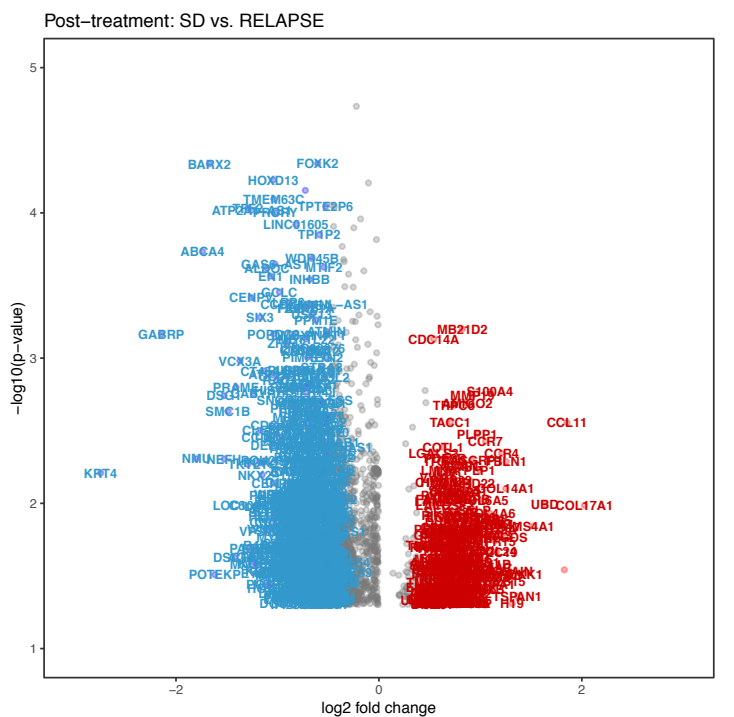

log2 fold change 
FIGURE 2: Investigation of three response biologies in treated tissue.

a: Histopathology figures of changing biomarker with therapy. Paired samples before (TURBT) and after (Cystectomy) treatment. The panel on the left is a patient presenting an inflamed phenotype at baseline and who relapsed after surgery. The arrow indicates the increased FAP expression after treatment. The panel on the right is from another patient whose phenotype changed from excluded to inflamed. Arrows indicate the increased number of CD8 cells. No increase in FAP was seen. This patient achieved stable disease with therapy.

b: Expression of the three IHC biomarkers before and after therapy. Biomarkers samples included those where paired biopsies are available (CD8 [n=54], PD-L1[n=59], FAP[n=53]). Discrepancy in number of samples analyzed stems from the fact that pCR patients do not have sufficient tumor tissue for exhaustive biomarker analysis in all samples. Results show increased expression of all four biomarkers with treatment. $\left({ }^{\star *}: p<0.01 ;{ }^{* \star *}: p<0.001\right)$. Samples were obtained from TURBT, before treatment (PRE) and from cystectomy after treatment (POST).

\section{c: CD8, PD-L1, FAP expression in responding and relapsing tumors before and after therapy.} Biomarkers samples included those where paired biopsies are available (CD8 [ $n=54]$, PDL1 [ $n=59]$, FAP [n=53]). Responders focused on patients with MPR. Relapsing patients were those with either radiological relapse (according to RECIST v1.1.) or died of any cause. Statistical analysis included Wilcoxon matched-pairs signed-rank test to compare Baseline and Cystectomy samples and Wilcoxon rank-sum test to compare the progressors and responders. Results showed increased CD8 levels in responders while FAP increased in relapsed patients $\left(^{* *}: p<0.01\right)$.

\section{d: Association between Lund molecular taxonomy subtypes and response to treatment ${ }^{19}$.}

Baseline (left panel) and post (right panel)-treatment samples were analyzed by response to treatment. Changes to molecular subgroup occurred in the majority of patients. Responding patients show a significant increase in the infiltrated group $(p<0.05)$ with a reduction in all other types. Patients with stable disease also show a less marked increase in the infiltrated type. No apparent difference occurs with the relapsed group. Analysis of the pCR patients included tissue from the tumor bed as no viable 
cancer cells were apparent. These responding patients (MPR,pCR) showed features of tissue repair within the infiltrated subgroup.

\section{e: Reactome analysis of differentially expressed genes between pre and post treatment time} points, for all response groups combined. The top 10 enriched pathways post-treatment (red) and pre-treatment (blue) were selected for display on the bar chart. PD-L1 blockade broadly associates with increases in stromal signatures (ECM, collagens) and decreases proliferation signature. P-values are given on the $x$ axis. . *: $p<0.05 ;{ }^{* *} p<0.01$

f: Correlation with TMB and outcome in pre and post treated samples. Treatment was not associated with any significant change in TMB, regardless of outcome group. TMB could not be assessed in responders as tumor DNA was absent.

g - h: Volcano plot and Reactome gene signature analysis for individual gene or genes signatures correlating with progression vs stable disease in treated samples. The reactome plot shows significant increased cell cycle signatures associated with relapsed sample (MKI67, CCNE1, BUB1, BUB1B, CCNB2, CDC25C, CDK2, MCM4, MCM6, MCM2). P-values are given on the $x$ axis. 
Figure 3

a RESPONSE Signature

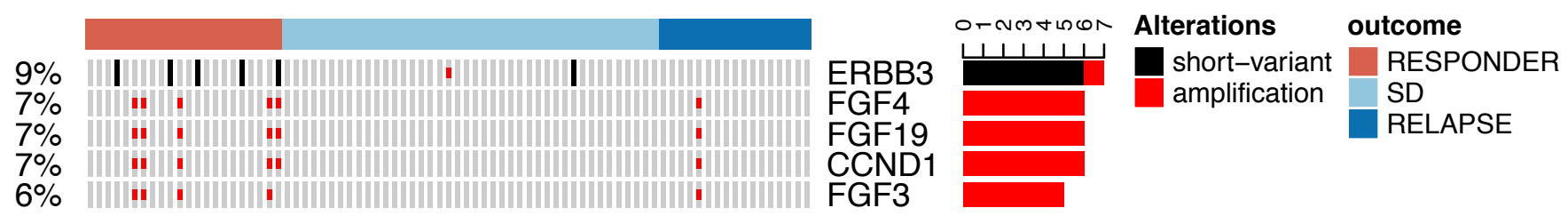

b

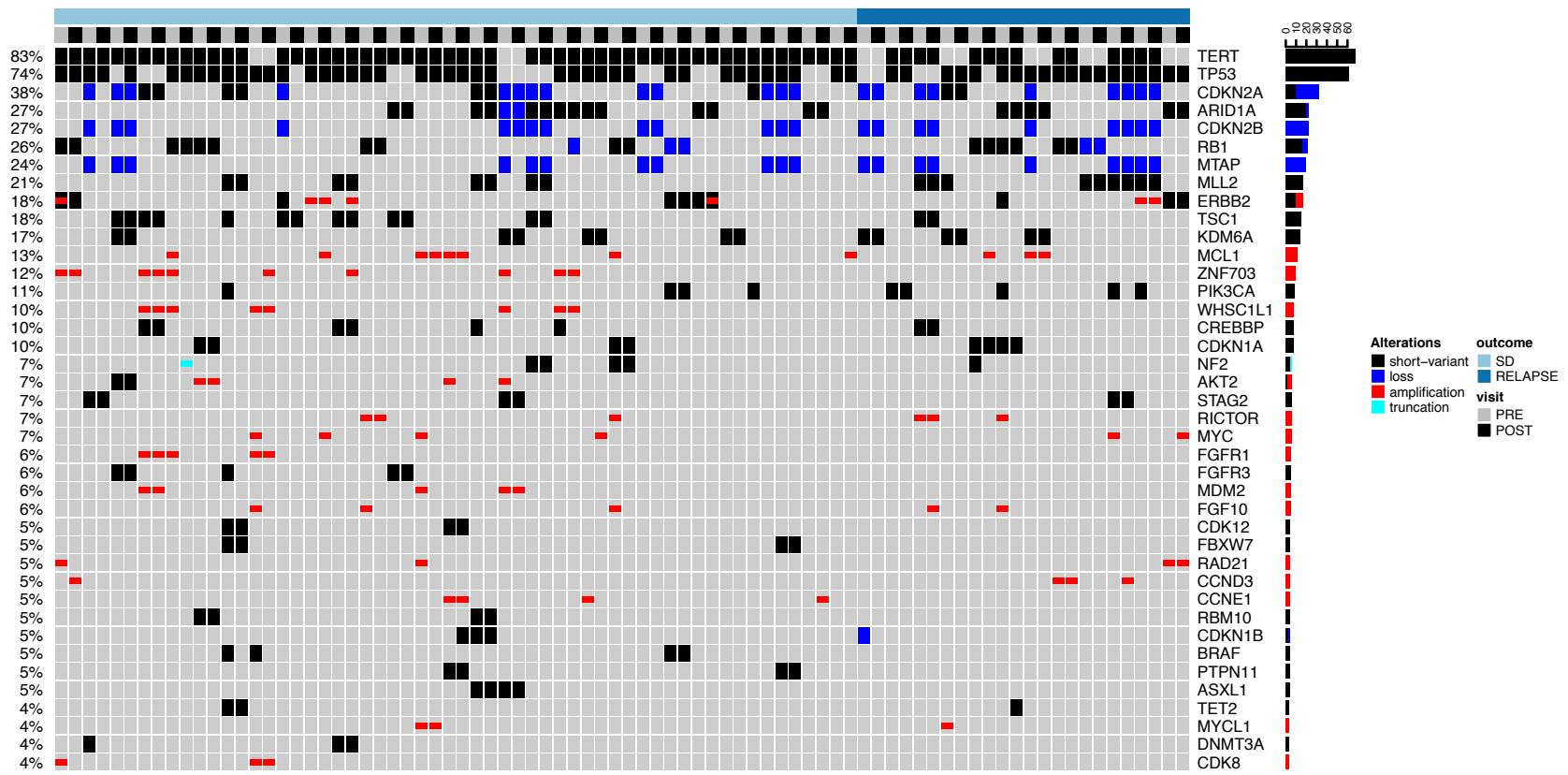

C

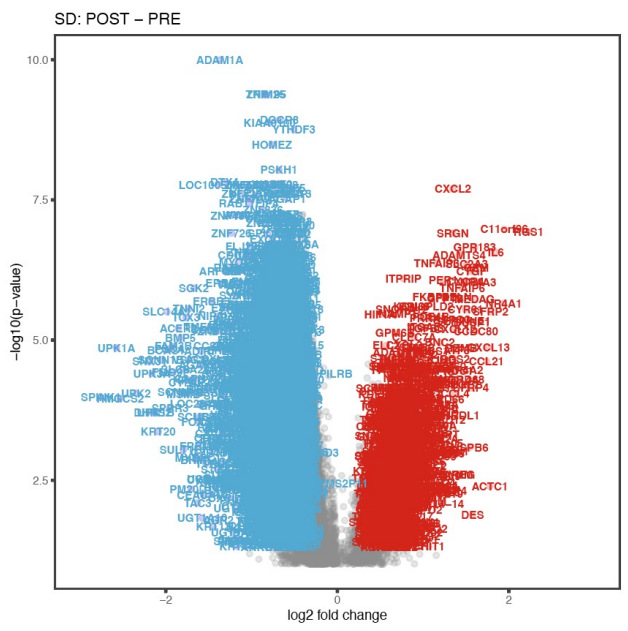

e

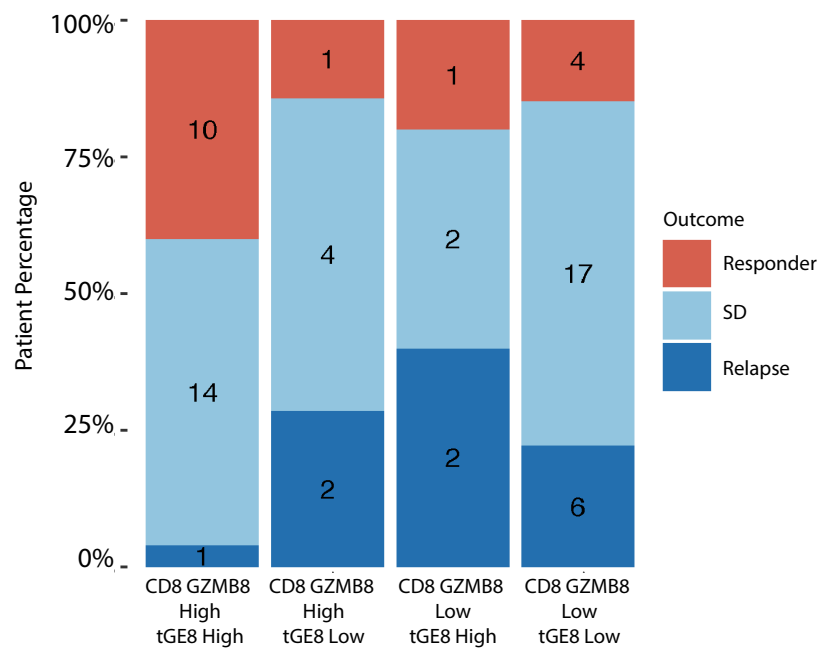

d SD_POSTVSPRE

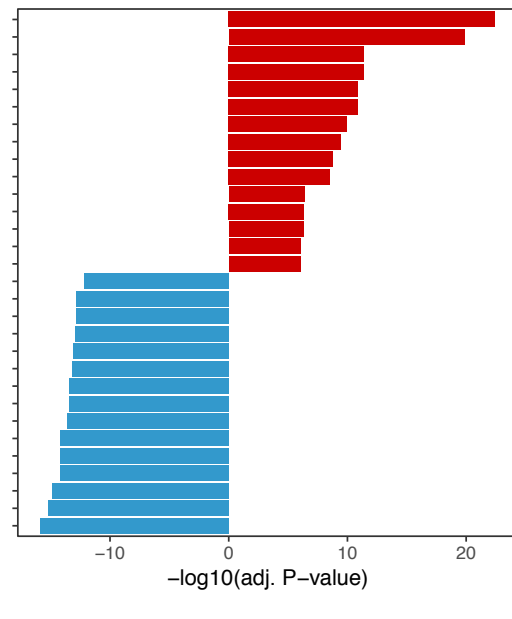

Extracellular matrix organization nterleukin-4 and Interleukin-13 signaling Interleukin-10 signaling Collagen formation Degradation of the extracellular matrix

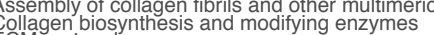
ECM proteoglycans Collagen chain trimerization Defective BJGALTL causes Peters-plus syndrome (PpS Mitochondrial translation initiation mRNA Splicing - Major Pathway Mitochondrial translation Respiratory electron transport, ATP synthesis by $\mathrm{c}$
he citric acid (TCA) cycle and respiratory electr (1) ranscriptional Regulation by TP53 Processing of Capped Intron-Containing Pre-mRNA
Translation

Down in SD-POST Up in SD-POST 
FIGURE 3

a: Oncoprint representing known DNA alterations from baseline tissue associated with response to neo-adjuvant atezolizumab therapy. Significance was assessed by chi-square test $(p<0.05)$, with alterations in 8 genes demonstrating an association with response, SD or relapse outcomes. 5 genes were specifically altered in responders, including ERBB3, FGF4, FGF19, CCND1 and FGF3.FGFR4. FGFR19, CCND1 and FGF3 (associated with response) are co-amplified since they are on the same locus: human Ch 11q13.3.

b: Oncoprint showing alterations before and after two cycles of therapy in patients with stable disease or relapse. Responding patients were not included due to absence or limited amount of cancer tissue in these samples. No consistent alterations occurred with therapy. This suggests that treatment outcome is not linked to expansion/shrinkage of specific cancer clones.

c: Volcano plots depicting differentially expressed genes between pre and post- treatment samples in stable disease patients. Genes were colored in red $(\log F C \geq 0.25)$ or blue $(\log F C \leq-0.25)$ if FDR pval $<0.05$. Comparative analysis was performed in this stable population because it was not influenced by response and relapse effects on gene expression. P-values are given on the $x$ axis.

d: Bar-chart representing geneset enrichment from the Reactome in pre- (blue) and post-(red) treatment samples in individuals with stable disease. Response and relapse patients were excluded to reduce tumor influences. The top 15 gene sets enriched in pre- or post-treatment samples were represented. A number of immune related genes were up-regulated. P-values are given on the $x$ axis.

e: Outcome of patients with high expression of the T-effector gene signature described in figure 1c and dual CD8/GZMB+ve staining. Enrichment for responders (40\%) and low relapse rates (4\%) can be seen. $P$ values are not given due to the exploratory analysis. 


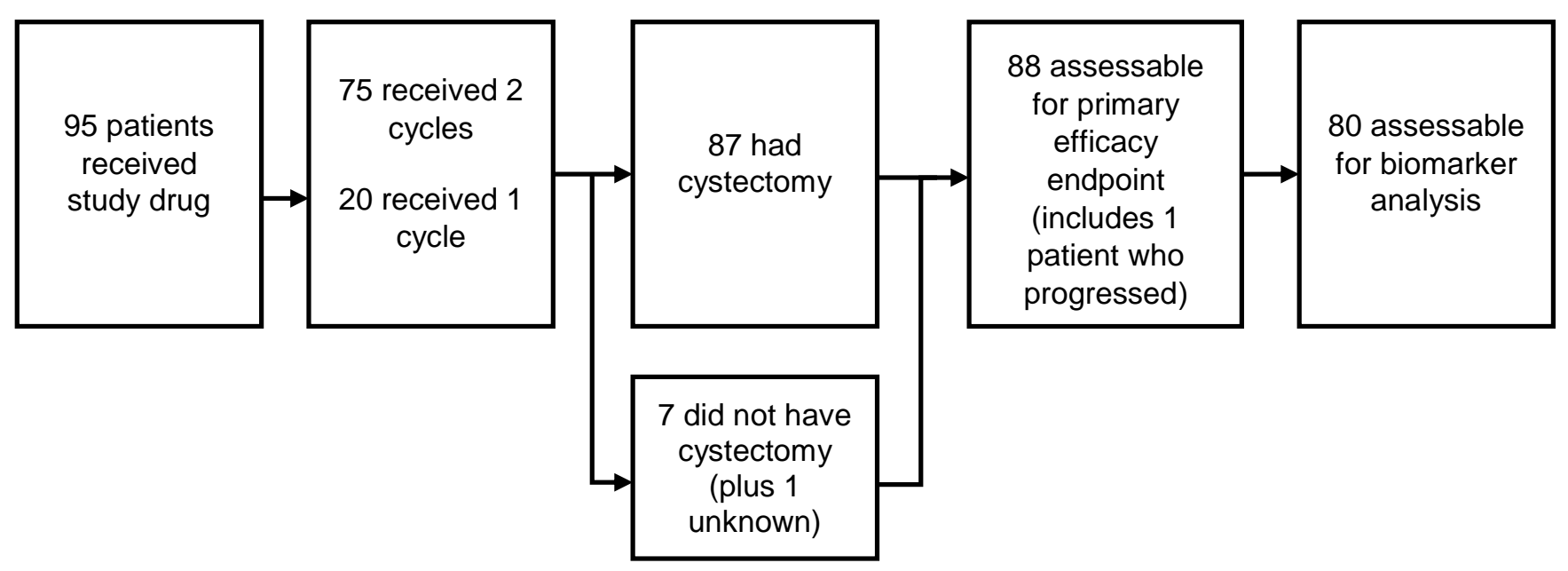

Extended Data Figure 1: Consort diagram. 95 patients were recruited to this study and received treatment. 75 received the full treatment regime with 2 cycles of atezolizumab and 20 patients were treated with only one cycle. In total, 87 patients underwent radical cystectomy, 7 patients did not undergo surgery and one patient withdrew consent from the study. 88 patients were assessable for the primary efficacy endpoint analysis, including one patient who experienced progression of disease. 


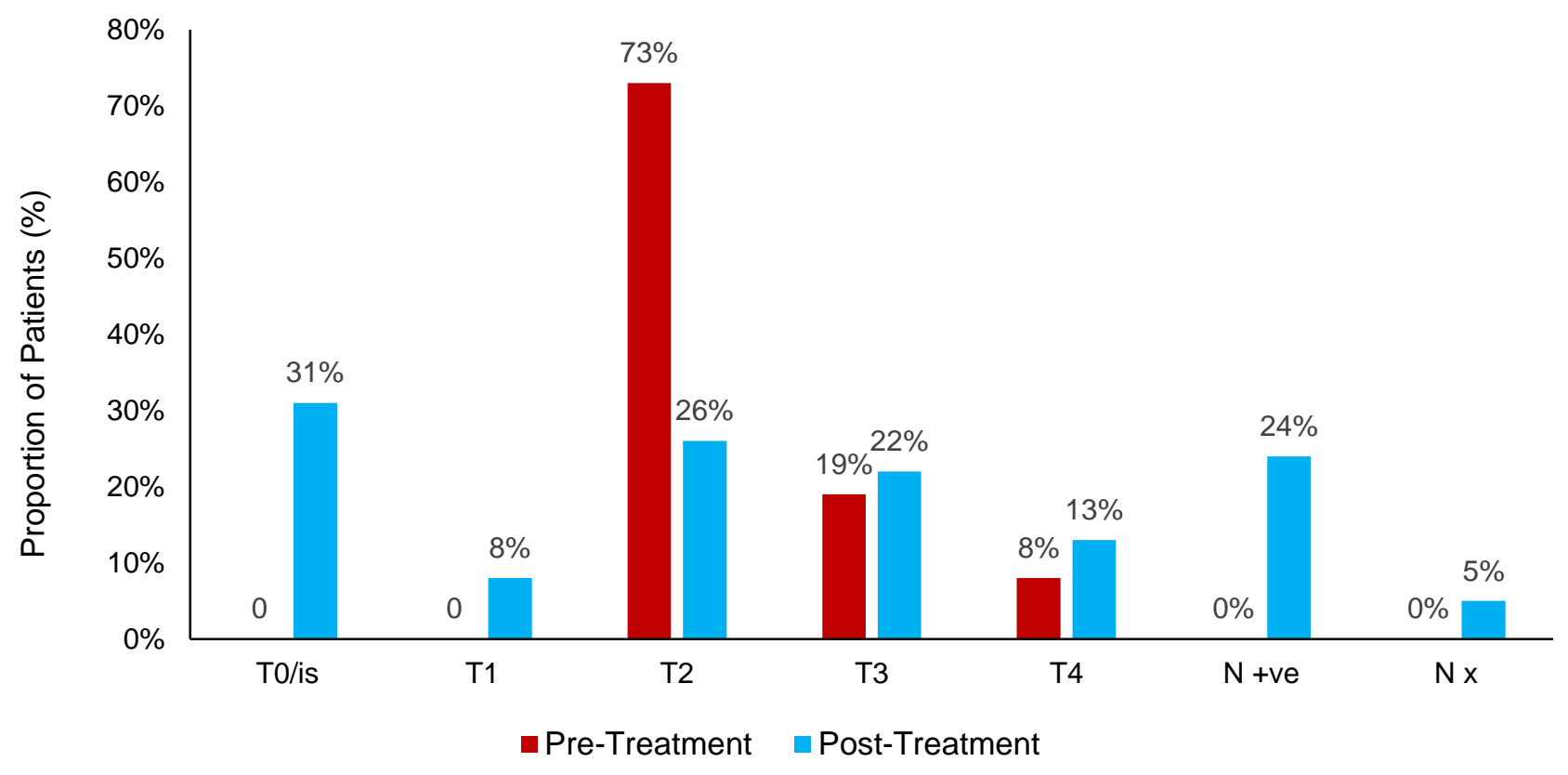

Extended Data Figure 2: Change in $\mathrm{T}$ and $\mathrm{N}$ stage associated with therapy. $\mathrm{T}$ and $\mathrm{N}$ stage at baseline was assessed with pathology samples from TURBT and cross-sectional imaging. $\mathrm{T}$ and $\mathrm{N}$ stage at surgery was assessed with pathology results from cystectomy and lymphadenectomy $(n=88)$. Direct comparisons between time points should be avoided due to differences in methodologies of assessment. Baseline nodal staging was radiological while it was pathological at surgery which may account for discrepancies. 


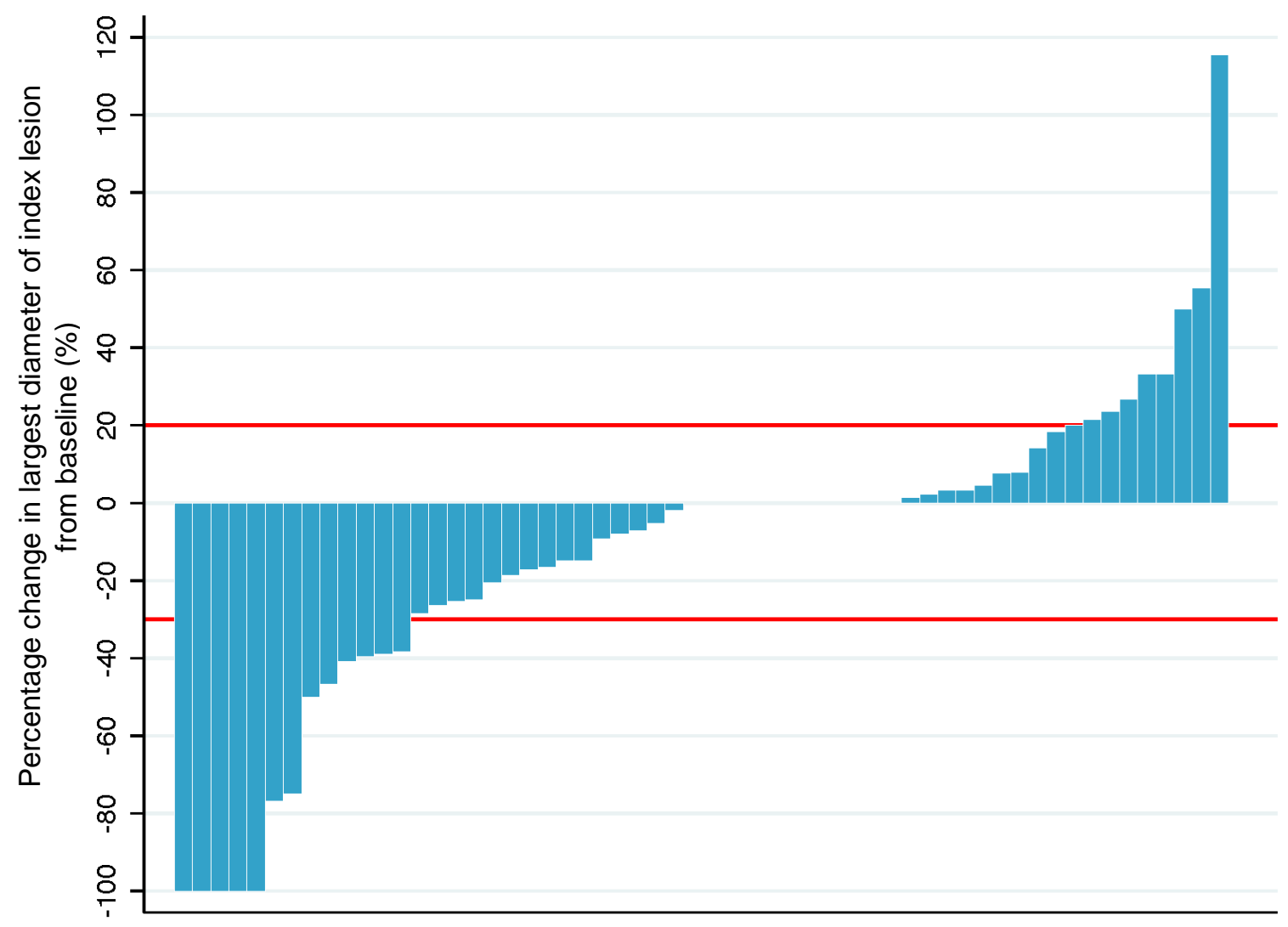

Extended Data Figure 3: Change in the size of bladder mass on cross sectional imaging. Imaging occurred at baseline and after completion of treatment prior to planned surgery. All patients were included irrespective if they were fit for surgery. Due to definitions of measurable disease not all bladder tumors were measurable at baseline. RECIST v1.1 was used to define response. A positive change denotes an increase in tumor size over time and conversely a negative change denotes a decrease in tumor size over time. Reference lines have been added for response (-30\% reduction in tumor size) and progression ( $20 \%$ increase in tumor size). 58 of the 95 treated patients had sequential imaging and radiologically measurable disease at baseline. Radiological progression (95\% $\mathrm{Cl})=9 / 58=16 \%(7-27)$. Radiological response $(95 \% \mathrm{Cl})=13 / 58=22 \%(13-35) .12$ patients exhibited no change, were not evaluable at pre-cystectomy scan, handled as $<10 \mathrm{~mm}$ at baseline, and therefore not included in this plot. 


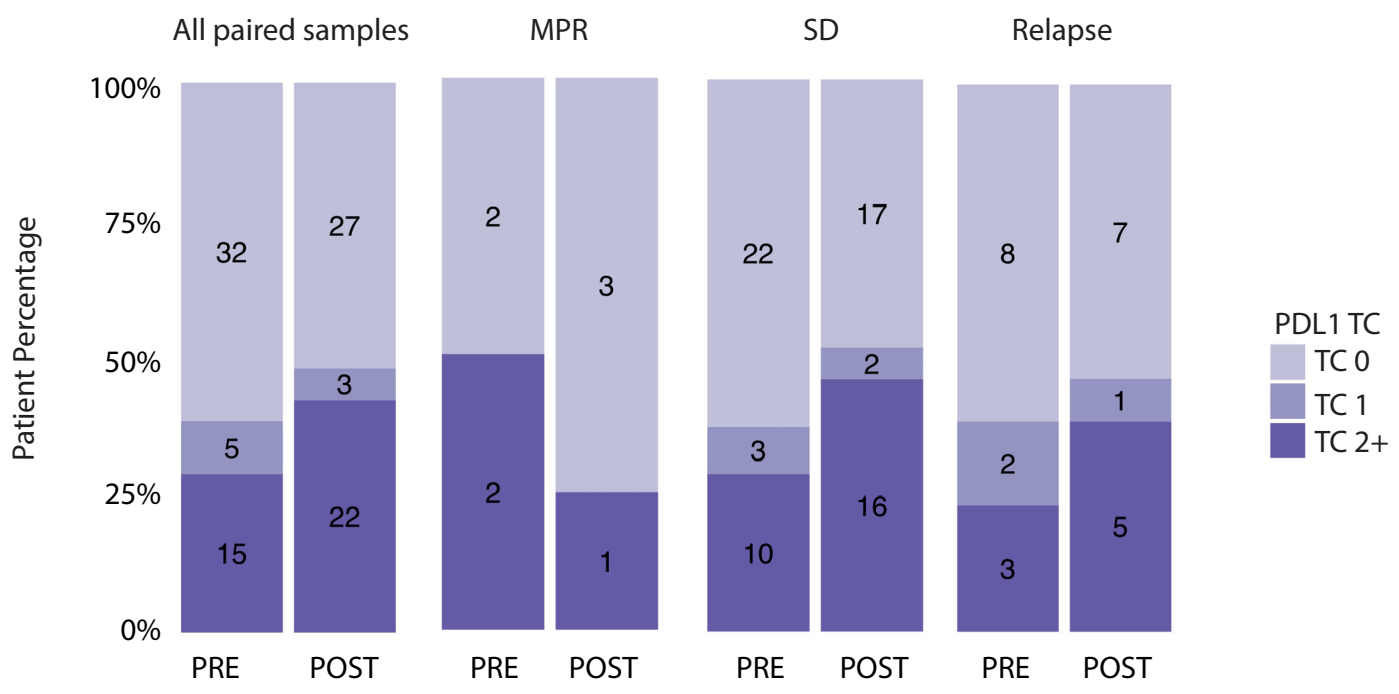

Extended Data Figure 4: Bar charts representing PD-L1 expression in tumor cells, in all samples or by outcome group. Only patients with measurable pre / post biopsies were considered in this analysis. 


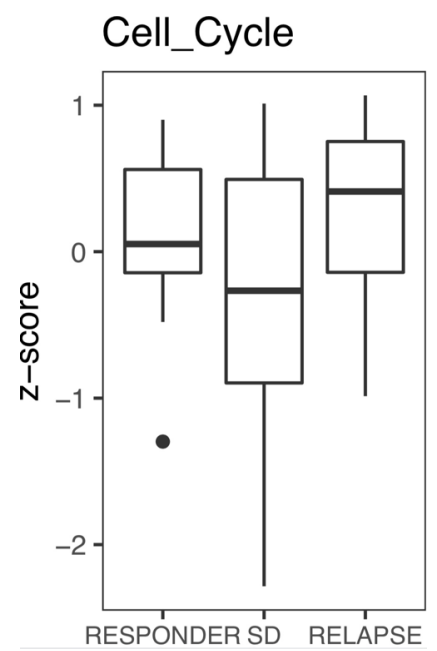

Extended Data Figure 5: Association between clinical outcome and the cell cycle signature at baseline. The cell cycle signature is calculated as the mean Z-score of the following genes: MKI67, CCNE1, BUB1, BUB1B, CCNB2, CDC25C, CDK2, MCM4, MCM6, MCM2. Median IQRs and ranges are shown. There was no significant difference between response and relapse samples. 


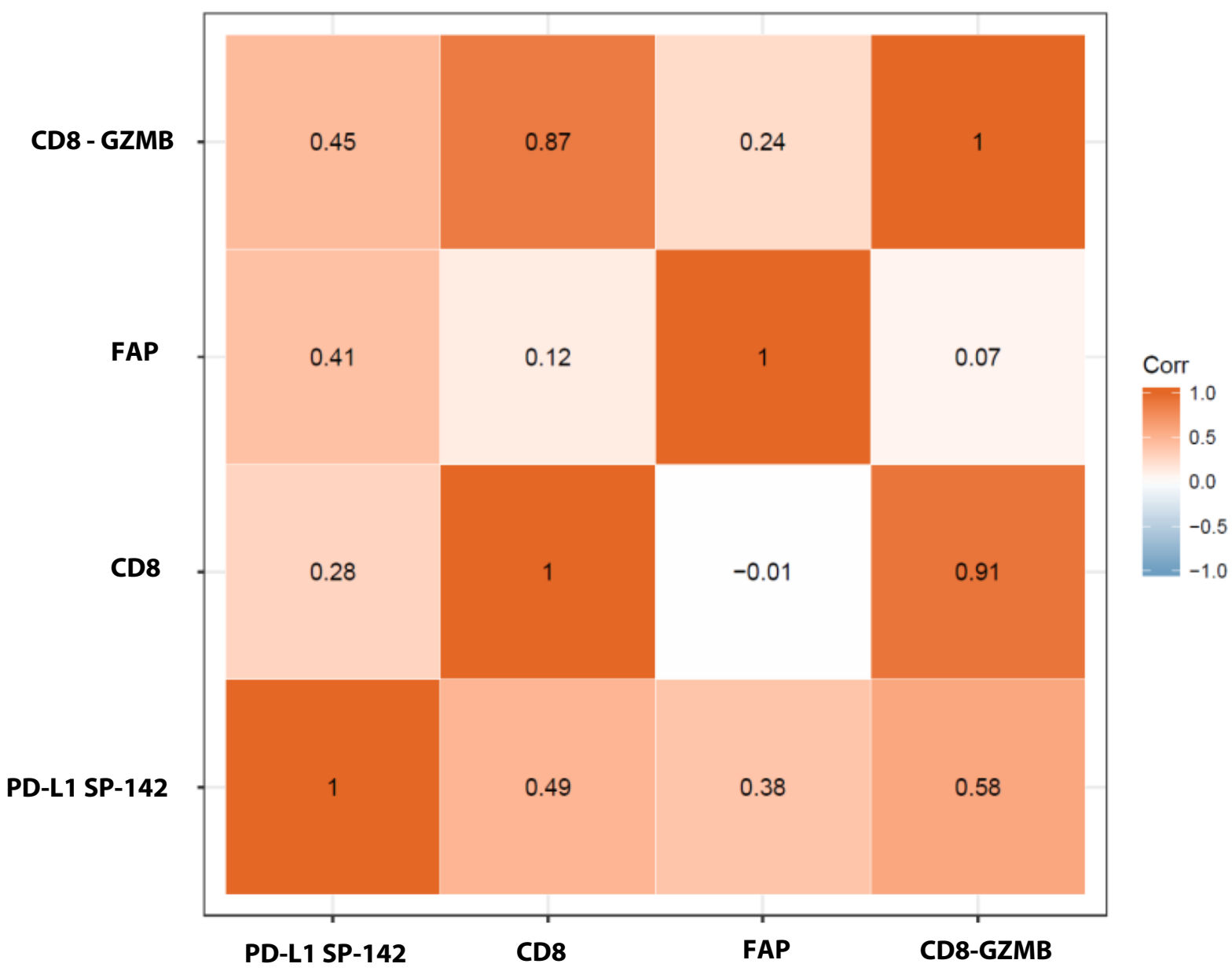

Extended Data Figure 6 Shows correlation between biomarkers. The expression 4 biomarkers were correlated with one another in terms of expression. Correlations were measured using Pearson's product moment correlation coefficient. The grid is split in the top left hand corner, which shows correlation in baseline samples and bottom right corner which shows correlation is treated samples. The darker the red the more significant the association. Most biomarkers correlated positively with one another. For consistency we present in treated and untreated samples. There was no correlation between FAP and CD8. Strongest correlation was between CD8-GZMB and PD-L1. 


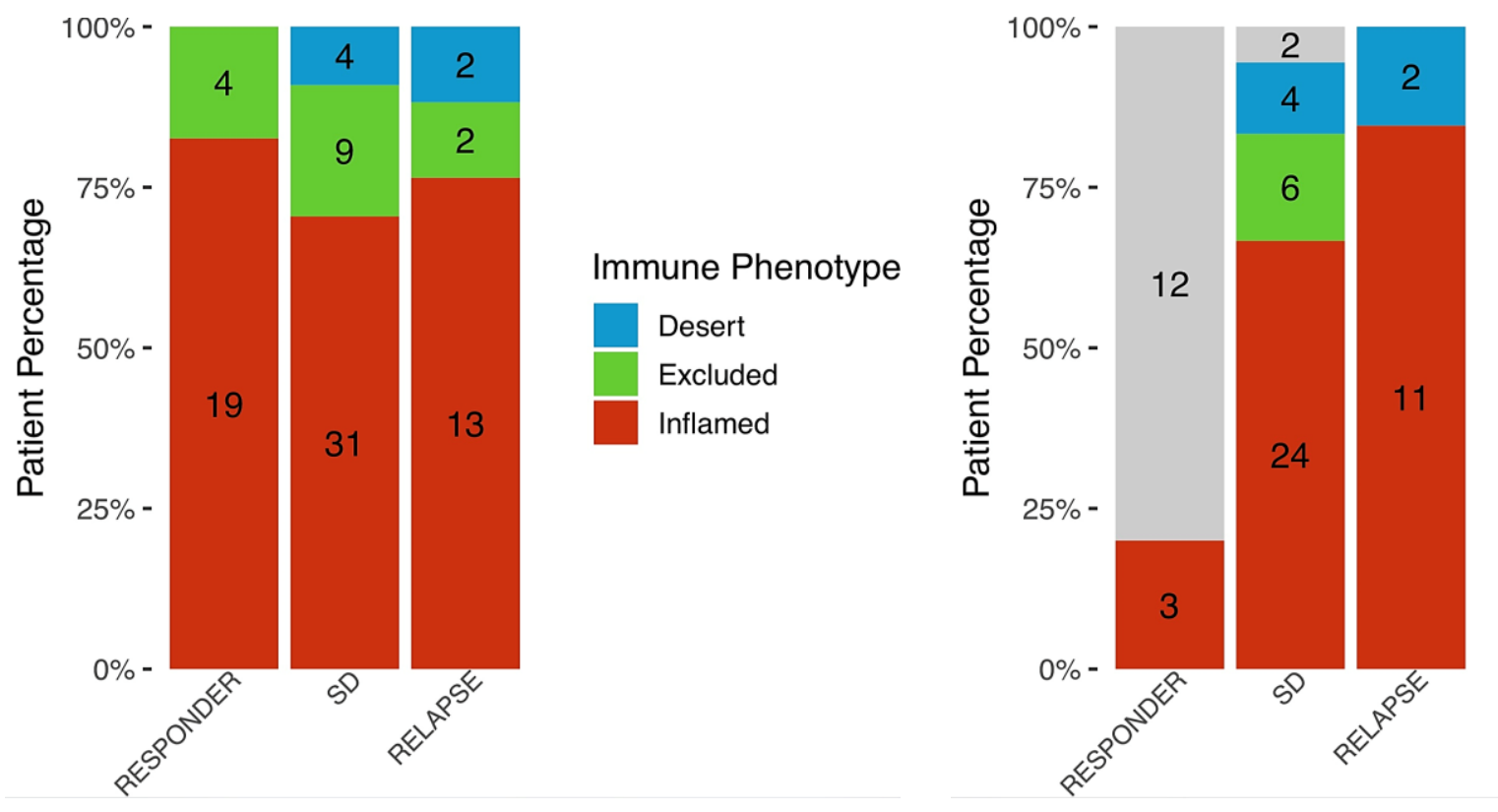

Extended Data Figure 7: Association between immune phenotypes and response to treatment. The frequency of immune phenotypes pre (left panel) and post (right panel) therapy by clinical outcome group. Immune phenotypes were assessed centrally by two pathologists using established methods ${ }^{11}$. 78 baseline and 57 cystectomy samples were assessed. A higher than expected proportion displayed inflamed phenotype compared to patients with metastatic disease. Deserts were not present in responding patients. Analysis of pCR samples was not possible because of lack of tumor cells. 


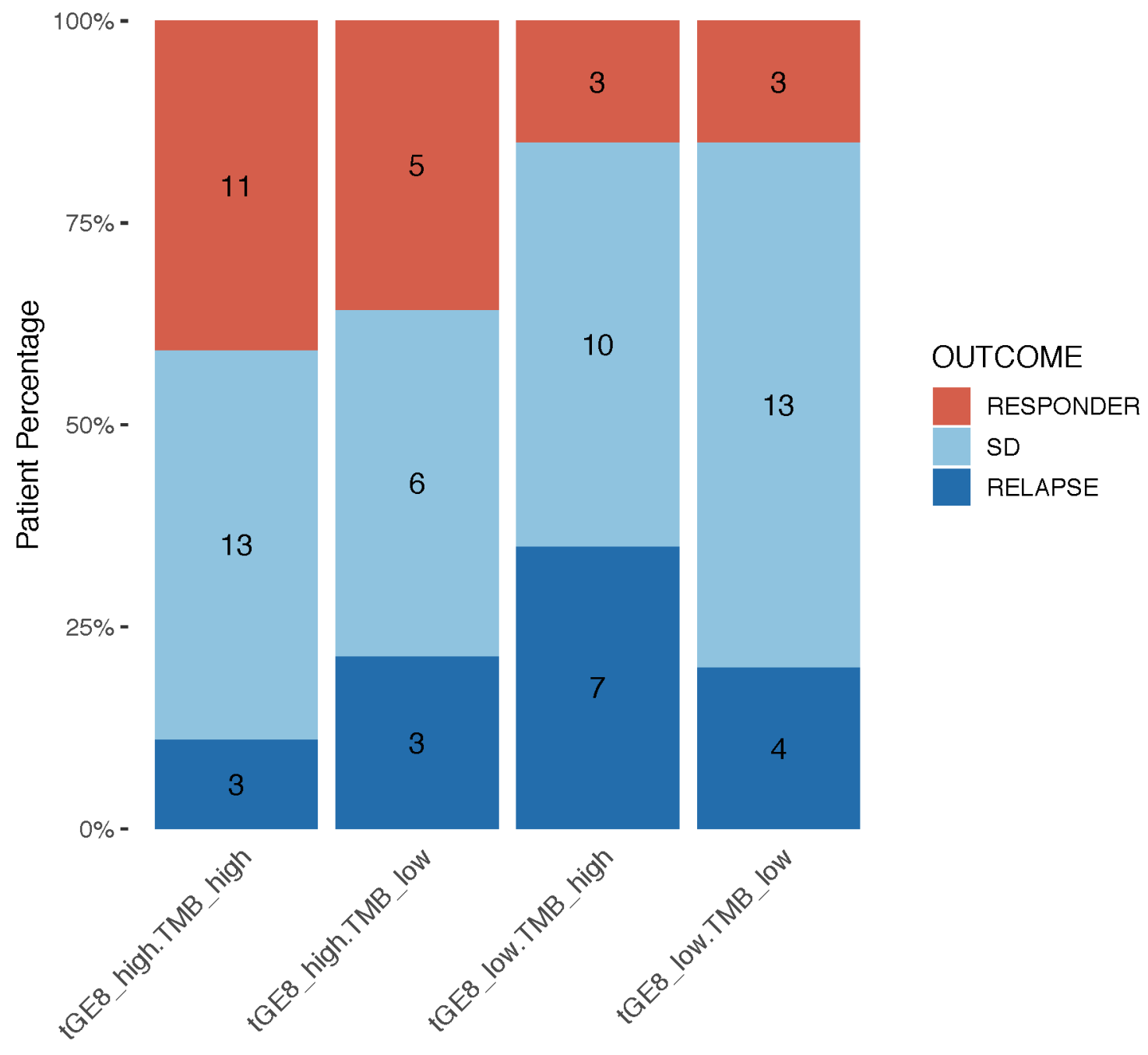

Extended figure 8: Tumors with dual high expression of T-effector signature (gene expression) and high TMB (Foundation medicine). Pre-treated tissue was taken for analysis. High levels were set as above median for both. No enrichment for response is seen for the addition of TMB. Previous data in the metastatic setting shows these 2 parameters enrich for response $\mathrm{e}^{3,13,15}$. 
Extended Data Table 1: Adverse events associated with atezolizumab.

\begin{tabular}{|c|c|c|c|c|}
\hline & $\begin{array}{c}\text { Grade 1-2 } \\
n\end{array}$ & $\begin{array}{c}\text { Grade 3-4 } \\
n\end{array}$ & $\begin{array}{c}\text { Grade } 5 \\
n\end{array}$ & $\begin{array}{c}N=95 \\
n\end{array}$ \\
\hline Patients with at least one related $A E$ & & & & 49 \\
\hline Fatigue & 18 & 2 & 0 & 20 \\
\hline Anorexia & 7 & 1 & 0 & 8 \\
\hline Transaminitis & 3 & 4 & 0 & 7 \\
\hline Pruritus & 7 & 0 & 0 & 7 \\
\hline Diarrhoea & 6 & 0 & 0 & 6 \\
\hline Rash & 6 & 0 & 0 & 6 \\
\hline Pyrexia & 4 & 1 & 0 & 5 \\
\hline Anaemia & 2 & 1 & 0 & 3 \\
\hline Headache & 3 & 0 & 0 & 3 \\
\hline Dyspnoea & 1 & 0 & 1 & 2 \\
\hline Hypertension & 1 & 1 & 0 & 2 \\
\hline Arthralgia & 2 & 0 & 0 & 2 \\
\hline Constipation & 2 & 0 & 0 & 2 \\
\hline Dry mouth & 2 & 0 & 0 & 2 \\
\hline Hypophosphataemia & 2 & 0 & 0 & 2 \\
\hline Infusion related reaction & 2 & 0 & 0 & 2 \\
\hline Mucositis & 3 & 0 & 0 & 3 \\
\hline Myositis & 2 & 0 & 0 & 2 \\
\hline Oedema & 2 & 0 & 0 & 2 \\
\hline CPK increased & 0 & 1 & 0 & 1 \\
\hline Myocarditis & 0 & 1 & 0 & 1 \\
\hline Nephritis & 0 & 1 & 0 & 1 \\
\hline Wound dehiscence & 0 & 1 & 0 & 1 \\
\hline Abnormal loss of weight & 1 & 0 & 0 & 1 \\
\hline Ageusia & 1 & 0 & 0 & 1 \\
\hline ALP increased & 1 & 0 & 0 & 1 \\
\hline Blood creatinine increased & 1 & 0 & 0 & 1 \\
\hline Bronchitis & 1 & 0 & 0 & 1 \\
\hline Buccal mucosal roughening & 1 & 0 & 0 & 1 \\
\hline Conjunctivitis allergic & 1 & 0 & 0 & 1 \\
\hline Dyspepsia & 1 & 0 & 0 & 1 \\
\hline Dysphonia & 1 & 0 & 0 & 1 \\
\hline Dysuria & 1 & 0 & 0 & 1 \\
\hline Hyperamylasaemia & 1 & 0 & 0 & 1 \\
\hline Hyperglycaemia & 1 & 0 & 0 & 1 \\
\hline Hyperproteinaemia & 1 & 0 & 0 & 1 \\
\hline Hypothyroidism & 1 & 0 & 0 & 1 \\
\hline Lung infection & 1 & 0 & 0 & 1 \\
\hline Lymphocyte count increased & 1 & 0 & 0 & 1 \\
\hline Nausea & 1 & 0 & 0 & 1 \\
\hline Pain & 1 & 0 & 0 & 1 \\
\hline Parasthesia & 1 & 0 & 0 & 1 \\
\hline Platelet count increased & 1 & 0 & 0 & 1 \\
\hline Thrombocytopenia & 1 & 0 & 0 & 1 \\
\hline Urinary tract infection & 1 & 0 & 0 & 1 \\
\hline Vomiting & 1 & 0 & 0 & 1 \\
\hline Weight decreased & 1 & 0 & 0 & 1 \\
\hline
\end{tabular}

